\title{
Modulation of the attentional blink by on-line response selection: Evidence from speeded and unspeeded Task ${ }_{1}$ decisions
}

\author{
PIERRE JOLICOEUR \\ University of Waterloo, Waterloo, Ontario, Canada
}

\begin{abstract}
Two critical target stimuli $\left(\mathrm{T}_{1}\right.$ and $\left.\mathrm{T}_{2}\right)$ were embedded in a stream of white letters shown on a black background, using a rapid serial visual presentation paradigm (RSVP, $100 \mathrm{msec} / \mathrm{item}$ ). $\mathrm{T}_{1}$ was a red $\mathrm{H}$ or $\mathrm{S}$; $\mathrm{T}_{2}$ was an $\mathrm{X}$ or a Y. Performance in a two-alternative discrimination on $\mathrm{T}_{2}$ was impaired when processing of $T_{1}$ was required-a result often called an attentional blink (AB). In previous work, the response in Task ${ }_{1}$ has been an unspeeded and delayed response at the end of the trial. Three experiments

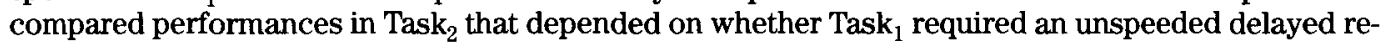
sponse or a speeded immediate response. A larger $\mathrm{AB}$ was found when a speeded response was re-

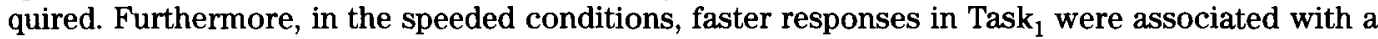
smaller and shorter $\mathrm{AB}$ effect than were slower responses. The results show that manipulations affecting a relatively late stage of processing - response selection-affect the magnitude and duration of the $\mathrm{AB}$ phenomenon. A new central inhibition theory is proposed to account for these results. According to this theory, the $\mathrm{AB}$ is similar to the psychological refractory period effect and is caused by central postponement of short-term consolidation of $\mathrm{T}_{2}$.
\end{abstract}

This article focuses on the effects of requiring a speeded response to the first of two critical stimuli in the attentional blink (AB) paradigm. Using this technique, the relationship between the duration of processing in Task and performance in Task ${ }_{2}$ can be investigated in a more direct way than in previous investigations of the $\mathrm{AB}$ phenomenon. The next section describes one of the paradigms that has been used to study the $\mathrm{AB}$ phenomenon, and the following sections describe four theoretical accounts designed to explain the principal features of the phenomenon. These sections are followed by a discussion of the potential effects of requiring a speeded response in Task ${ }_{1}$ in the usual paradigm used to study the AB phenomenon. Three experiments are then presented. In these experiments the effects of speeded versus delayed Task response requirements are compared. The results show that the nature of the response requirements in Task ${ }_{1}$ has systematic effects on the shape of the $\mathrm{AB}$ function, as does

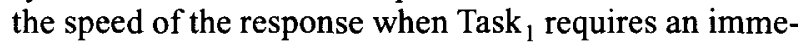
diate response. The implications of the results are discussed in the General Discussion section.

\section{The Attentional Blink Phenomenon}

Several researchers have demonstrated a strikingly large and long-lasting deficit in the ability to perform a simple

This work was supported by a Research Grant from the Natural Sciences and Engineering Research Council of Canada. I thank Margaret Ingleton for technical assistance and Jim Johnston, Rob McCann, Ritske De Jong, Kim Shapiro, Molly Potter, Gordon Logan, and David Irwin for helpful comments and criticisms of this work. Correspondence concerning this article should be sent to P. Jolicoeur, Department of Psychology, University of Waterloo, Waterloo, ON N2L 3G1, Canada (e-mail: pjolicoe@,cgl.uwaterloo.ca). judgment on a second critical stimulus, $T_{2}$, when this judgment follows another stimulus, $T_{1}$, that also requires a simple judgment. For example, Raymond, Shapiro, and Arnell (1992, Experiment 2) presented letters, using rapid serial visual presentation (RSVP) at the same location on the screen, with $90 \mathrm{msec}$ separating the onsets of consecutive letters. The letters were black, except for $T_{1}$, which was white. There were two blocks of trials that differentiated the experimental from the control condition. In the experimental condition, there were two tasks: Task and Task . Task $_{1}$ was to report the identity of $\mathrm{T}_{1}$ (the white letter). Task ${ }_{2}$ was to report whether an $\mathrm{X}$ had also been shown in the RSVP stream after $T_{1}$. The responses in both tasks were made at the end of the trial, without speed pressure, after the termination of the RSVP stream. $T_{1}$ was presented in every trial, whereas $\mathrm{T}_{2}$ (black $\mathrm{X}$ ) was presented in half of the trials. In the control condition, only Task ${ }_{2}$ was performed, and subjects were asked to ignore $\mathrm{T}_{1}$.

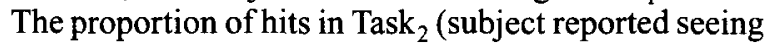
an X when one was shown) for each position of $T_{2}$ in the RSVP stream relative to the position of the white letter is plotted in Figure 1. In the control condition, performance was uniformly high across the positions in the RSVP stream in which $T_{2}$ could occur, with the highest performance being when $\mathrm{T}_{2}$ itself was white (position 0 ) or when it was the last letter in the stream (position +8 ). Nothing followed the last position in the RSVP stream, so this item was not subject to backward masking, which accounts for the upswing in performance relative to position +7 in both the control and experimental conditions. In contrast, accuracy (the proportion of hits) in the experimental condition was much lower for positions +2 to +6 , although remaining roughly equivalent to that in the control con- 


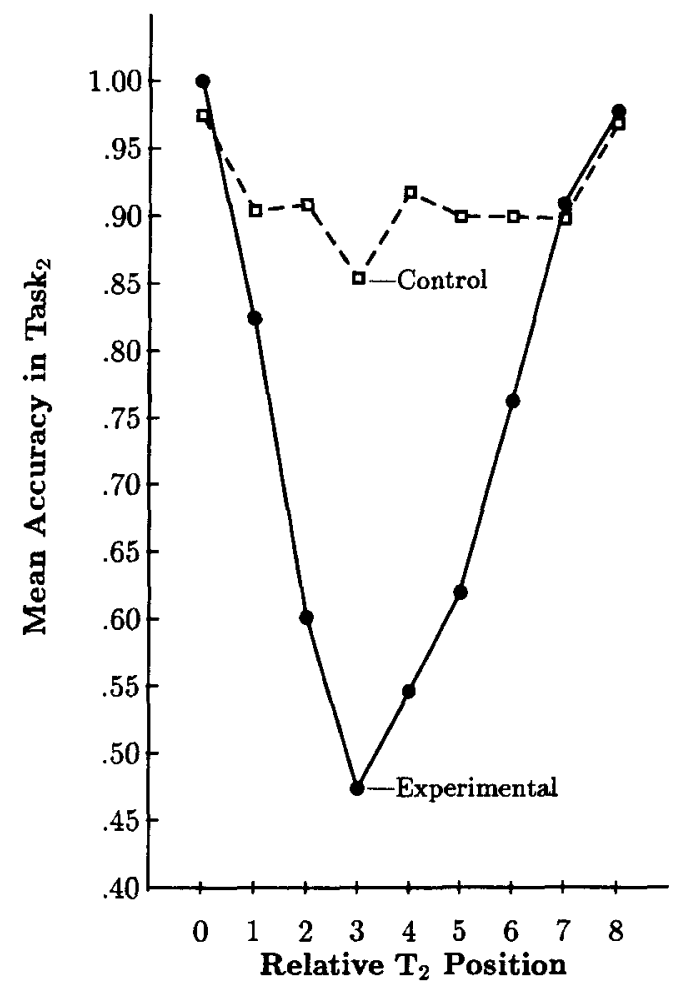

Figure 1. Results from Raymond, Shapiro, and Arnell (1992), Experiment 2. Mean proportion correct report of $T_{2}$ on $T_{2}$-present (X-present) trials. (Estimated from their figure.)

dition for relative stream positions $0,+1,+7$, and +8 . The performance deficit in Task 2 in the experimental condition relative to the control condition at the intermediate stream positions following $T_{1}$ was called an attentional blink (AB) by Raymond et al. (1992). Several other researchers have reported similar findings in paradigms that were significantly different (see, e.g., D. E. Broadbent \& M. H. P. Broadbent, 1987; Chun \& Potter, 1995; Weichselgartner \& Sperling, 1987), attesting to the robustness of the effect.

\section{Models of the Attentional Blink Phenomenon}

The sections below contain a brief review of four models that have been proposed to account for the results of the $\mathrm{AB}$ phenomenon as found in the type of paradigm described in the previous section.

Attentional gate model. Raymond et al. (1992) proposed that the processing of $T_{1}$ in the experimental condition of the experiment described in the previous section begins with a preattentive detection of the white letter. This preattentive detection was postulated to initiate an attentional response, leading to the identification of $T_{1}$. The identification of $T_{1}$ involves the opening and closing of an attentional gate whose purpose is to regulate the flow of visual information to pattern-recognition centers of the brain (see Weichselgartner \& Sperling, 1987). Raymond et al. (1992) hypothesized that the at- tentional gate would close for a longer time when information following $T_{1}$ could be potentially confused with $T_{1}$. The perception of $T_{2}$ suffers if it is presented while the attentional gate is closed. "In the blink analogy, the locking of the gate is like the closing of an eyelid" (Raymond et al., 1992, p. 859).

Similarity theory. Shapiro, Raymond, and Arnell (1994) proposed an account of the AB phenomenon that is different from the attentional gate model account of 1992 (see also Raymond, Shapiro, \& Arnell, 1995). They revised their position on the basis of results from experiments in which $T_{1}$ was a period of time during the RSVP stream in which there was no patterned stimulus (achieved by replacing a letter in the stream by a blank field). They found a very much reduced $A B$ effect, despite the fact that Task ${ }_{1}$ was quite difficult to perform (as indexed by error rates in Task ${ }_{1}$ ) relative to other experiments producing a substantial $\mathrm{AB}$ effect.

Shapiro et al. (1994) and Shapiro and Raymond (1994) interpreted the small $\mathrm{AB}$ effect in the temporal gap experiments as an indication that visual patterned stimulation in the $T_{1}$ stimulus is a necessary condition for the manifestation of an $\mathrm{AB}$. This led them to propose an alternative theory - similarity theory - that has three components. First, there is an early and parallel stage of visual information processing that produces representations of items in the visual field. Second, a selection template for $T_{1}$ (and $T_{2}$ ) is matched against the representations produced by the first stage. Third, items that match the template for $T_{1}$ and $T_{2}$ are selected for entry into visual short-term memory (VSTM), which has limited capacity. Items that gain entry into VSTM are assigned weights that determine the probability of report from VSTM. The similarity between distractor items (most importantly the item following $T_{1}$ ) and the selection template for $T_{1}$ (and between the item following $T_{2}$ and the template for $T_{2}$ ) is an important determinant of entry into VSTM. When similarity is low, distractors are less likely to match the selection template and thus less likely to gain access to VSTM. Shapiro et al. argued that, as a result of temporal limitations in the speed of selection, the item immediately following $T_{1}$ and the item immediately following $T_{2}$ both have a high likelihood of gaining entry into VSTM. The weights assigned to items in VSTM also depend on a pool of limited resources. Items that enter VSTM earlier are assigned larger weights, all else being equal, because there are more available resources. Later items receive smaller weights, because resources have been depleted by the entry of earlier items in VSTM.

The probability of report from VSTM at the end of a trial is determined by weights that are assigned to each item in VSTM. A higher weight results in a higher probability of output from VSTM. The weights are a function of the goodness of match to the selection templates. Although distractors, in general, will have lower weights than $T_{1}$ and $T_{2}$, distractors that match the selection templates partially may nonetheless have a weight that is sufficiently 
high to result in performance decrements when items are selected for report from VSTM. Both the number of items in VSTM and their weights are postulated to influence performance. The AB effect, in this theory, occurs at the time of output from VSTM, as a function of the weights associated with the items in VSTM. In order to account for the recovery of performance in Task ${ }_{2}$ (the task associated with $T_{2}$ ) as the delay between $T_{1}$ and $T_{2}$ is increased, Shapiro and Raymond (1994) propose that $\mathrm{T}_{1}$ either decays or is flushed from VSTM in the $450 \mathrm{msec}$ following its presentation, so that $T_{1}$ no longer interferes with $T_{2}$. Presumably, $T_{1}$ has had time to be transferred from VSTM to another system (short-term memory [STM]?), but this is not made clear by Shapiro and Raymond.

Attentional dwell model. Duncan, Ward, and Shapiro (1994) and Ward, Duncan, and Shapiro (1996) proposed that attention should be thought of as a sustained state that is necessary in order to make representations of relevant objects available for guiding behavior. They contrast their theoretical position with the view that attention can proceed in a high-speed serial fashion, moving from item to item very quickly (say, $30 \mathrm{msec}$ per item, as might be suggested by visual search studies; see, e.g., Wolfe, 1994).

Ward et al. (1996) are admirably clear about what they believe should not cause an $\mathrm{AB}$ effect. They claim that the $A B$ effect is not due to perceptual masking, the number of object attributes identified, the number of responses made, limits in the number of locations that must be attended, or the particular details of their presentation methods. In their experiments, $T_{1}$ was a simple pattern (say, a digit) presented at one of two possible locations and followed by a pattern mask. $\mathrm{T}_{2}$ was another simple pattern (say, a letter) presented at one of two different locations and also followed by a mask. The stimulus onset asynchrony (SOA) between $T_{1}$ and $T_{2}$ was varied. Report of the identity of $T_{2}$ showed a pattern like the one displayed in Figure 1, whereas report of $T_{1}$ was generally good, regardless of SOA.

They proposed that the $\mathrm{AB}$ phenomenon is caused by a limited visual-processing capacity for encoding objects into a form that can make contact with other cognitive mechanisms. A greater demand on capacity is made when a larger number of objects is attended. One of their experiments provided good support for this last claim. Either one $\left(T_{1}\right)$ or two $\left(T_{1}\right.$ and $\left.T_{1}{ }^{\prime}\right)$ stimuli had to be encoded in a first observation interval, and, at a variable SOA from this event, a second observation interval presented another pattern $\left(T_{2}\right)$. Report of $T_{2}$ showed a larger $\mathrm{AB}$ effect when two patterns had to be reported from the first event than when only one had to be reported.

In this model, objects compete for a share of limited capacity visual-processing resources, according to their match to a target template. The competition for limited resources resolves gradually over several hundred milliseconds, and the winners engage visual-processing mechanisms at the expense of the losers. This theory makes the clear prediction that the response selection (RS) requirements of Task ${ }_{1}$ should not modulate the magnitude of the $\mathrm{AB}$ effect.
Two-stage model. Chun and Potter (1995) proposed a two-stage model for the $\mathrm{AB}$ phenomenon. In the first stage, called rapid detection, almost every item presented in RSVP streams at a rate of about 10 items/sec is identified and can be selected for access to processing in subsequent stages of processing. Stage 1 representations are subject to rapid forgetting when there is interference from subsequent items in the RSVP stream, unless they are selected for further processing.

In the second stage, called capacity-limited processing, items selected for further processing are transferred into a more durable representation (such as verbal STM). Stage 1 representations cannot serve as the basis for a later verbal report or for a manual response. The information must first be transferred to STM, which results in full identification and consolidation for subsequent report. Stage 2 processing is capacity limited and is initiated by a transient attentional response that occurs on Stage 1 detection of a probable target. The $A B$ phenomenon is caused by the capacity limit in Stage 2 processing. If $\mathrm{T}_{2}$ is presented while $T_{1}$ is processed in Stage 2, Stage 2 processing of $T_{2}$ must wait, and $T_{2}$ may be forgotten.

The central interference theory. Another account of the $\mathrm{AB}$ phenomenon, called the central interference the$o r y$, is described in the General Discussion section. Although this account was developed independently from the two-stage model, and from the perspective of postponement models of psychological refractory period (PRP) interference, it is similar to the model proposed by Chun and Potter (1995). Such convergence of views arrived at from different perspectives is encouraging. The main difference between the two-stage model and the central interference theory is that the latter makes an explicit identification between the dual-task interference observed in the PRP paradigm and that observed in the $A B$ paradigm. (The theory is presented in more detail in subsequent sections of the article.) The explicit identification of $A B$ interference with the type of interference believed to be the cause of the PRP phenomenon suggests that some (or all) of the AB effect should be caused by a relatively late, postperceptual, locus of dual-task interference, such as response selection (McCann \& Johnston, 1992; Pashler, 1994). The experiments in this article provide an initial investigation of this possibility.

\section{The Present Experiments}

The experiments presented below introduced a simple modification to the paradigms used to study the AB phenomenon to date. In some cases, Task ${ }_{1}$ required an immediate and speeded response to $T_{1}$ rather than a delayed response at the end of the trial. The purpose of the empirical work was twofold. One was to discover whether requiring an immediate response to $T_{1}$ would have any impact on the magnitude and shape of the $\mathrm{AB}$ effect. The second was to discover whether there would be a relationship between response times (RTs) in Task ${ }_{1}$ and the magnitude of the AB effect.

If the $\mathrm{AB}$ and PRP phenomena are produced by fundamentally different causes - with $A B$ reflecting an ear- 
lier stage of processing, such as a late stage of stimulus encoding, and PRP reflecting a later stage of processing, such as response selection - one might expect that the response requirements of Task ${ }_{1}$ would have little impact on the results in the AB paradigm. In this view, changing the response requirements of Task ${ }_{1}$ would affect stages of processing taking place after the processes involved in the AB effect have already run to completion.

On-line versus off-line processing of $T_{1}$. The most obvious consequence of requiring a speeded response in Task $_{1}$ is to force a temporal overlap between operations associated with response-related stages of processing required to perform Task ${ }_{1}$, such as response selection, and the operations required to perform Task ${ }_{2}$. That is, response selection must be made on line in this case. When the response to $T_{1}$ is delayed, operations like selecting a response can take place off line, long after the time-critical processing for Task ${ }_{2}$ has already taken place. The AB effect is a processing deficit observed in Task ${ }_{2}$ performance. In order to investigate whether a relatively late process, such as response selection, has any potential involvement in the $\mathrm{AB}$ effect, one must create a situation in which there is at least a reasonable possibility of some temporal overlap between the operations required to perform Task $_{2}$ and these later stages of processing for Task ${ }_{1}$. If an immediate response must be made to $\mathrm{T}_{1}$, operations like response selection cannot be deferred to a later time (such as the end of the trial). These operations must be performed soon after the presentation of $T_{1}$, while the processing of $\mathrm{T}_{2}$ may be proceeding concurrently. Thus, the delayed-response paradigm may be inherently ill suited to investigate the question of whether response selection can influence the critical process (or processes) producing the $\mathrm{AB}$ phenomenon. This could explain why some previous work has failed to observe interactions between response-level manipulations and the $\mathrm{AB}$ phenomenon (see, e.g., Ward et al., 1996).

The foregoing arguments suggest that one effect (perhaps the principal effect) of requiring an immediate and speeded response in Task k $_{1}$ is to increase the likelihood of a temporal overlap between postperceptual processing, such as response selection, and the processes required to perform Task $\mathrm{T}_{2}$. This is why the manipulation of the nature of the response in Task ${ }_{1}$ - delayed versus speededis important. If this manipulation has an obvious effect on the magnitude of the $A B$ effect, it will be difficult to escape the conclusion that the causal mechanism(s) mediating the $\mathrm{AB}$ phenomenon cannot be uniquely associated with relatively early perceptual capacity limitations.

The foregoing sections described four models (other than the central interference theory) designed to account for the AB phenomenon. None of these models predicts that requiring an immediate response in Task ${ }_{1}$ should have an effect on the magnitude, duration, or shape of the $A B$ function. Ward et al. (1996) were the clearest on this point in that their attentional dwell model explicitly excludes late processes, such as those associated with response selection, as being causally involved in the $A B$ phenomenon. Furthermore, none of the other three models - the attentional gate model (Raymond et al., 1992), the similarity theory (Shapiro et al., 1994), or the two-stage model (Chun \& Potter, 1995) - has an obvious way to relate the $\mathrm{AB}$ phenomenon to mechanisms, such as response selection, that might come into play when a speeded response is required. All of these models assume that the locus of the $\mathrm{AB}$ effect takes place in stages of processing that occur before response selection. Showing that a manipulation of the response requirements in Task (immediate vs. delayed) modulates the magnitude of the $A B$ phenomenon would suggest either that the locus of the $A B$ effect is later in processing than has been supposed so far or that there are multiple loci (e.g., one early and one late).

In this article, the theoretical issue of the relationship between the $A B$ phenomenon and response selection is operationalized in terms of two empirical issues. The first is the manipulation of the response requirements associated with Task -namely, whether the response in Task $_{1}$ $\left(R_{1}\right)$ is immediate or delayed. As the experiments will show, the magnitude of the $A B$ effect is larger when $R_{1}$ is performed on-line than when $R_{1}$ is performed off line. The introduction of speeded Task ${ }_{1}$ responses also introduces new possibilities for data analysis. In particular, it allows us to measure on-line processing time across various manipulations in Task ${ }_{1}$ and trial-to-trial variation in

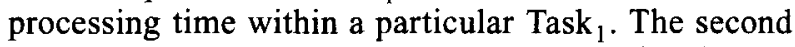
empirical issue is related to these trial-to-trial variations in processing time in Task $\mathrm{x}_{1}$. The main finding here is that the magnitude of the $\mathrm{AB}$ effect increases as $\mathrm{RT}_{1}$ becomes longer. The implications of these findings is considered in the General Discussion section.

\section{GENERAL METHOD}

This section contains an outline of the methods that were common across the experiments. The reader can assume that the method was as specified here unless a specific mention of a difference is noted in the section describing a particular experiment.

\section{General Stimuli}

The stimuli were uppercase letters, presented in white on a black background, on an SVGA color computer screen controlled by a 386 , 486 , or 586 CPU. The letters were presented in RSVP, at the same location at the center of the screen, at a rate of 10 letters/sec. Each letter was exposed for $100 \mathrm{msec}$ with no blank interstimulus interval. These parameters are identical to those used by Chun and Potter (1995) and thus are known to create conditions under which an AB can be observed.

There were 6-9 letters (this number selected randomly at run time) presented prior to $T_{1}$ and 9-12 (also randomized at run time) following $T_{1}$. $T_{2}$ could occur in any of the eight positions following $T_{1}$ with equal probability. Thus, even in the last position for $T_{2}$, there were between 1 and 4 letters following $T_{2}$, which means that the effective masking conditions required to observe an $\mathrm{AB}$ in Task $_{2}$ were always in effect (Giesbrecht \& Di Lollo, in press). 
On every trial, the background stream items were selected at random, without replacement, from the letters of the alphabet, excluding $\mathrm{H}, \mathrm{S}, \mathrm{X}$, and $\mathrm{Y}$.

The letters subtended about $1^{\circ}$ of visual angle. White letters had an approximate luminance of $25 \mathrm{~cd} / \mathrm{m}^{2}$ and $\mathrm{CIE}(x, y)$ coordinates of $(.278, .306)$, as measured by a CS-100 Minolta meter. Red letters had an approximate luminance of $26 \mathrm{~cd} / \mathrm{m}^{2}$ and $\operatorname{CIE}(x, y)$ coordinates of $(.355,286)$. The background was black with a luminance of less than $1 \mathrm{~cd} / \mathrm{m}^{2}$. These values are approximate, because the experiments were conducted on several computer systems; average values are reported above. Small variations in these parameters are not likely to be important. Note that the luminances of white and red letters were very similar, effectively ruling out the possibility that $\mathrm{T}_{1}$ would mask $\mathrm{T}_{2}$ more than would distractor stimuli.

\section{General Procedure}

A pair of symbols (e.g., ++ ) was presented at the center of the screen, in green, at the beginning of each trial. The symbols provided feedback on performance in the previous trial and acted as a fixation point in the current trial. The left member of the pair indicated performance in Task ${ }_{1}$, the right member in Task ${ }_{2}$. A + sign indicated a correct response and a - sign indicated an error. The fixation symbols for the first trial in a block were always pluses, so there was no feedback for the last trial in a block of trials.

Each trial was initiated by pressing the space bar on the computer keyboard, which caused the fixation/feedback symbols to disappear and the onset of the RSVP stream.

The critical stimulus for Task $\mathrm{K}_{1}, \mathrm{~T}_{1}$, was a red letter that could be either an $\mathrm{H}$ or an $\mathrm{S}$. $\mathrm{T}_{1}$ was presented on half of the trials and not presented on the other half, with these two types of trials occurring equally often but intermixed at random within each block of trials. $\mathrm{T}_{1}$-present trials were thus the experimental trials. $\mathrm{T}_{1}$-absent trials were used as within-session within-subjects control trials in which no AB should occur because there was no stimulus to trigger Task . $_{1}$ These trials provided a good control for dual-task costs of maintaining the processes required to perform Task in $_{1}$ a prepared state (De Jong \& Sweet, 1994; Pashler, 1994). This type of control trial does not control for potential sensory masking effects of $T_{1}$ on $T_{2}$ However, considerable prior work shows that such masking does not occur, even when $T_{1}$ is considerably more luminous than $T_{2}$ (see, e.g., Raymond et al., 1992).

Task , was to discriminate between $\mathrm{H}$ and $\mathrm{S}$ when a red letter was shown. If no red letter was shown, the space bar was to be pressed at the end of the RSVP sequence, without speed pressure. When a

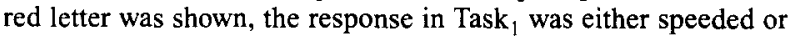
delayed. When delayed, the program requested a response for Task at the end of the trial. When speeded, a response was expected as quickly as possible, while the remainder of the RSVP stream was displayed. The .> key was to be pressed for $\mathrm{H}$ or the /? key for $\mathrm{S}$. Both of these response keys were at the lower right of the keyboard, and the index and middle fingers of the right hand could rest comfortably on them during a block of trials. The same response keys were used in every experiment.

In every experiment, one of the eight positions following $T_{1}$ in the RSVP sequence contained $T_{2}$, which was always either an $X$ or a $Y$. The streams for $T_{1}$-absent trials were created in the same way as in $T_{1}$-present trials, except that $T_{1}$ was replaced by a randomly selected distractor letter. This procedure allowed the stream positions in which $T_{2}$ was shown to be labeled in the same way across $\mathrm{T}_{1}$-present and $\mathrm{T}_{1}$-absent trials.

Task $_{2}$ was to decide whether $T_{2}$ was an $X$ or a $Y$, and subjects knew that one or the other was always shown. Task ${ }_{2}$ was unspeeded in all cases. The response for Task ${ }_{2}$ was always performed after the response for Task $\mathrm{T}_{1}$. The $\mathrm{X}$ key on the keyboard was used to indicate the presence of an $\mathrm{X}$; the $\mathrm{C}$ key was used to indicate the presence of a $\mathrm{Y}$.
The SOA between $T_{1}$ (or the position $T_{1}$ would have occupied when $T_{1}$ was not shown) and $T_{2}$ was $100,200, \ldots, 700$, or 800 msec. Each subject performed 640 experimental trials ( 10 blocks of 64) following 64 practice trials ( 2 blocks of 32 ). Each block of experimental trials had an equal number of trials for each combination of $\mathrm{T}_{1}$-present/absent, $\mathrm{H}$ versus $\mathrm{S}, \mathrm{X}$ versus $\mathrm{Y}$, and SOA.

\section{General Method of Analysis}

Because of the rapid presentation rate of the stimuli in the RSVP streams, some subjects found it difficult to perform Task ${ }_{2}$, even when $T_{1}$ was not presented, resulting in performance constrained by a floor effect. The following procedure was adopted to deal with this problem. First, the mean performance in Task ${ }_{2}$ in the $T_{1}$-absent condition was computed for each subject, pooling trials from all SOAs. Any subject whose performance fell below $69 \%$ correct was excluded from further analyses. In analyses of Task ${ }_{2}$ performance (including the screening procedure described above), only trials with correct responses in Task , were included. Given that Task $_{1}$ performance was very good, this conditional analysis excluded few trials. Analyses that included the rejected subjects, error trials in Task $_{1}$, or both produced patterns of results that were very similar to the ones that are reported below, so these procedures did not distort the empirical picture.

The results for Task $_{2}$ were based on the proportion of correct trials. These proportions were usually submitted to analyses of variance (ANOVAs) in which SOA (8) and the presence/absence of $\mathrm{T}_{1}$ were within-subjects factors. These ANOVAs were used to estimate $95 \%$ within-subjects confidence intervals (Loftus \& Masson, 1994), which are given in the graphs displaying the results of each separate experiment, as opposed to graphs produced to compare results across experiments. In these latter graphs, the error bars are $95 \%$ confidence intervals computed from separate ANOVAs performed on the results from each SOA (for $T_{1}$-present trials). Departures from these conventions are indicated where appropriate.

In the present experiments, the $\mathrm{AB}$ effect manifested itself as a deficit in performance in $\mathrm{Task}_{2}$ in the $\mathrm{T}_{1}$-present condition, relative to the $T_{1}$-absent condition, usually over the first $400 \mathrm{msec}$ following $T_{1}$. For SOAs ranging between 500 and $800 \mathrm{msec}$, performance across the $T_{1}$-present and $T_{1}$-absent conditions was more similar, suggesting that the blink was essentially over across these SOAs (for most trials for most subjects). These observations were used to create a method of analysis designed to estimate the magnitude of the $\mathrm{AB}$ effect that could also be used to compare the magnitude of the $\mathrm{AB}$ effect across conditions. The method consisted of taking the mean difference between the experimental condition ( $T_{1}$-present) and the control condition ( $\mathrm{T}_{1}$-absent) across the first four SOAs (100$400 \mathrm{msec})$, and again across the last four SOAs (500-800 msec). Because performance in the control condition ( $\mathrm{T}_{1}$-absent trials) was essentially unaffected by SOA (e.g., see Figures 2-3), the overall mean performance level, $\bar{C}$, for the control condition was computed by averaging the mean performance for each SOA for the $T_{1}$-absent condition:

$$
\bar{C}=\frac{\sum_{i=1}^{8} C_{i}}{8},
$$

where $C_{i}$ is the mean for the $i$ th SOA. In a typical analysis, each subject's data were first reduced to 16 means, 8 in the $T_{1}$-present condition ( 1 for each SOA) and 8 in the $T_{1}$-absent condition (again, 1 for each SOA). This procedure described above reduced these 16 means to just 2 : the mean difference between the $T_{1}$-present condition and the $T_{1}$-absent condition across the shorter SOAs, 


$$
A B_{1,4}=\frac{\sum_{i=1}^{4}\left(\bar{C}-E_{i}\right)}{4},
$$

and the mean difference between the $T_{1}$-present condition and the $\mathrm{T}_{1}$-absent condition across the longer SOAs,

$$
A B_{5,8}=\frac{\sum_{i=5}^{8}\left(\bar{C}-E_{i}\right)}{4},
$$

where $E_{i}$ is the mean for the experimental condition ( $\mathrm{T}_{1}$-present $)$ in the $i$ th SOA condition. A significantly larger number for the shorter SOAs than for the longer SOAs was expected when a condition produced an $\mathrm{AB}$ effect. For example, for the results shown in Figure 2, the mean $A B_{1,4}$ score was $.167 \pm .026$, and the mean $A B_{5,8}$ score was $.055 \pm .026$, as would be expected if there was a large deficit in Task ${ }_{2}$ performance immediately following $T_{1}$ and if this effect was attenuated at longer SOAs. The error estimates following the means, here and elsewhere in the article, are $95 \%$ confidence intervals for either within-subjects or between-subjects comparisons, as appropriate for the particular means and intended comparison (Loftus $\&$ Masson, 1994).

Differences in $A B$ magnitudes across conditions are revealed by interactions between the difference of the estimates of control minus experimental performance for shorter versus longer SOAs, with the variable defining the conditions under study.

Accuracy in Task ${ }_{2}$ is plotted as a function of the SOA between $T_{1}$ and $T_{2}$, expressed in units of deciseconds ( $1 \mathrm{dsec}=100 \mathrm{msec}$ ), or equivalently as a function of the position of $T_{2}$ in the RSVP stream, relative to the position of $T_{1}$ (relative $T_{2}$ position).

\section{General Subjects}

Most of the subjects were undergraduate students at the University of Waterloo who volunteered to participate for pay or for course credit. A few were graduate students or staff. All had normal or corrected-to-normal visual acuity, and all reported having normal color vision. Table 1 gives some statistics for each experiment.

\section{EXPERIMENT 1 \\ Task $_{1}$ Unspeeded, Task $2=X / Y$ 2AD}

The purpose of Experiment 1 was to establish the appropriateness of the general experimental methods described above, to replicate the $A B$ phenomenon, and to provide a comparison group for Experiment 2. $T_{1}$ was a red $\mathrm{H}$ or a red $\mathrm{S}$, and Task $\mathrm{k}_{1}$ required an unspeeded twoalternative discrimination ( $2 \mathrm{AD}$ ) between $\mathrm{H}$ and $\mathrm{S}$. $\mathrm{T}_{2}$ was an $\mathrm{X}$ or a $\mathrm{Y}$ in the same color as other distractor RSVP stream items. Task ${ }_{2}$ was an unspeeded $2 \mathrm{AD}$ between $\mathrm{X}$ or Y. Both responses were made after the end of the RSVP stream.

Table 1

Subject Information for Each Experiment

\begin{tabular}{ccccccc}
\hline Exp & $N$ & Males & Females & $N_{\text {rej1 }}$ & $N_{\text {rej2 }}$ & Age \\
\hline 1 & 19 & 7 & 12 & 4 & 0 & 20.1 \\
2 & 19 & 10 & 9 & 4 & 0 & 20.0 \\
3 & 22 & 7 & 15 & 6 & 1 & 20.6 \\
\hline
\end{tabular}

Note-Exp, experiment number; $N$, number of subjects in final sample, after rejection criteria; Males, males in final sample; Females, females in final sample; $N_{\text {rej }}$, rejected because Task ${ }_{2}$ accuracy was below .69 in control trials; $N_{\mathrm{rej}_{2}}$, rejected for responding after the end of the trial rather than on line; Age, mean age.

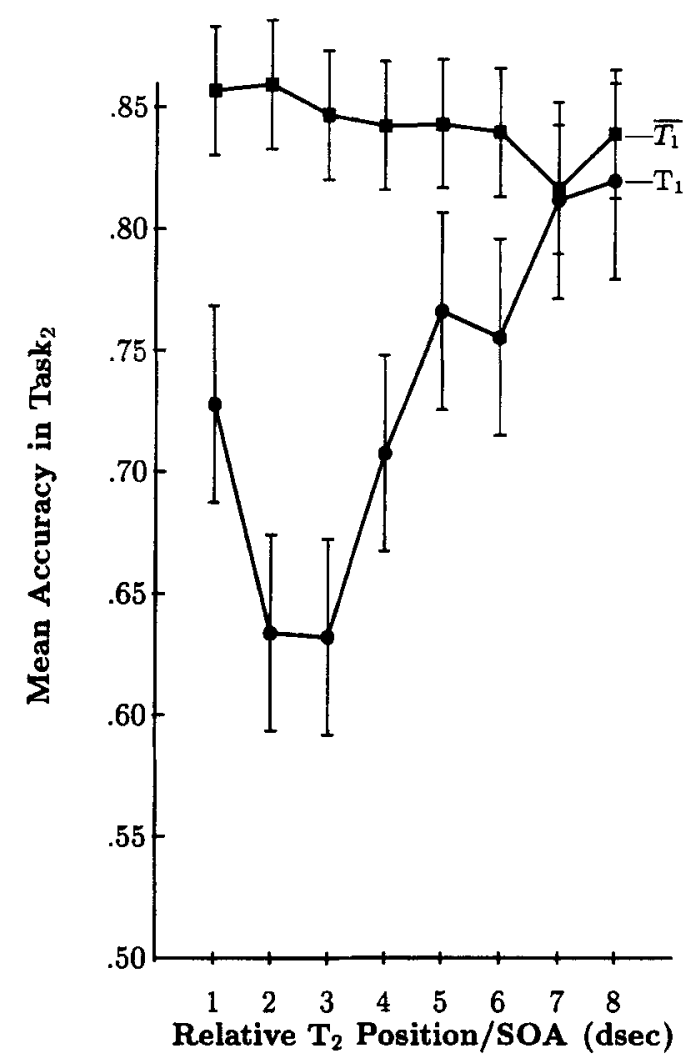

Figure 2. Results from Experiment 1. Mean proportion correct discrimination of $T_{2}\left(X\right.$ vs. $Y$ ) in $T_{1}$-absent trials (control trials, labeled $\bar{T}_{1}$ ) versus $T_{1}$-present trials (experimental trials, labeled $\left.T_{1}\right) .1$ decisecond (dsec) $=100 \mathrm{msec}$. The error bars are $95 \%$ within-subjects confidence intervals appropriate for comparisons of means across SOAs within each function $\left(T_{1}\right.$ or $\left.\overline{T_{1}}\right)$.

\section{Results and Discussion}

The mean proportion of correct responses in Task ${ }_{2}$ is shown in Figure 2 for each SOA between $T_{1}$ and $T_{2}$ and for trials in which there was a red letter $\left(T_{1}\right.$-present $)$ and trials in which there was no red letter $\left(\mathrm{T}_{1}\right.$-absent). On average, performance was $84 \%$ in the $T_{1}$-absent condition and did not differ appreciably across SOAs $(F<1$, in a separate ANOVA). In contrast, accuracy in the targetpresent condition displayed the classic $U$-shaped $\mathrm{AB}$ function, with a pronounced deficit at SOAs of 200-300 msec and a sharp recovery as SOA was increased.

The mean $A B_{1,4}$ score was $.167 \pm .026$, whereas the mean $A B_{5,8}$ score was $.055 \pm .026[F(1,18)=40.40$, $\left.M S_{\mathrm{e}}=0.002975, p<.0001\right]$, confirming the clear-cut and substantial $A B$ effect evident in the figure.

Performance in Task $_{1}$, for the $T_{1}$-present trials, was good, with a mean of $91.1 \%$ correct. Accuracy in Task did not depend on the SOA at which $\mathrm{T}_{2}$ was presented $(F<1)$.

These results demonstrated a large $\mathrm{AB}$ effect when Task $_{2}$ required a $2 \mathrm{AD}$ between an $\mathrm{X}$ and a $\mathrm{Y}$. The large and clear $A B$ effect found with this task validated the methodology used in Experiment 1 and provided a comparison group for the subjects tested in Experiment 2. 


\section{EXPERIMENT 2}

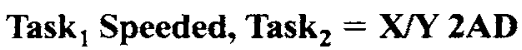

Experiment 2 was like Experiment 1, except that Task required a speeded on-line response. The purpose of the experiment was to compare the effects of requiring a speeded response in Task $_{1}$ with performance measured in

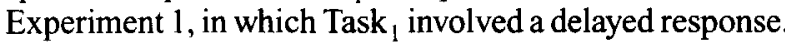

\section{Results and Discussion}

The mean proportion correct in Task ${ }_{2}$ is shown in Figure 3 for each SOA between $T_{1}$ and $T_{2}$ and for trials in which there was a red letter $\left(T_{1}\right.$-present $)$ and trials in which there was no red letter $\left(T_{1}\right.$-absent). On average, performance was $82 \%$ in the $\mathrm{T}_{1}$-absent condition and did not differ appreciably across SOAs $(F<1)$. In contrast, accuracy in the target-present condition displayed the classic $\mathrm{U}$-shaped $\mathrm{AB}$ function, with a pronounced deficit at SOAs of 200-300 msec and a sharp recovery as SOA was increased.

As expected from Figure 3, there was a large statistical difference between the $A B_{1,4}$ score $(.226 \pm .035)$ and the

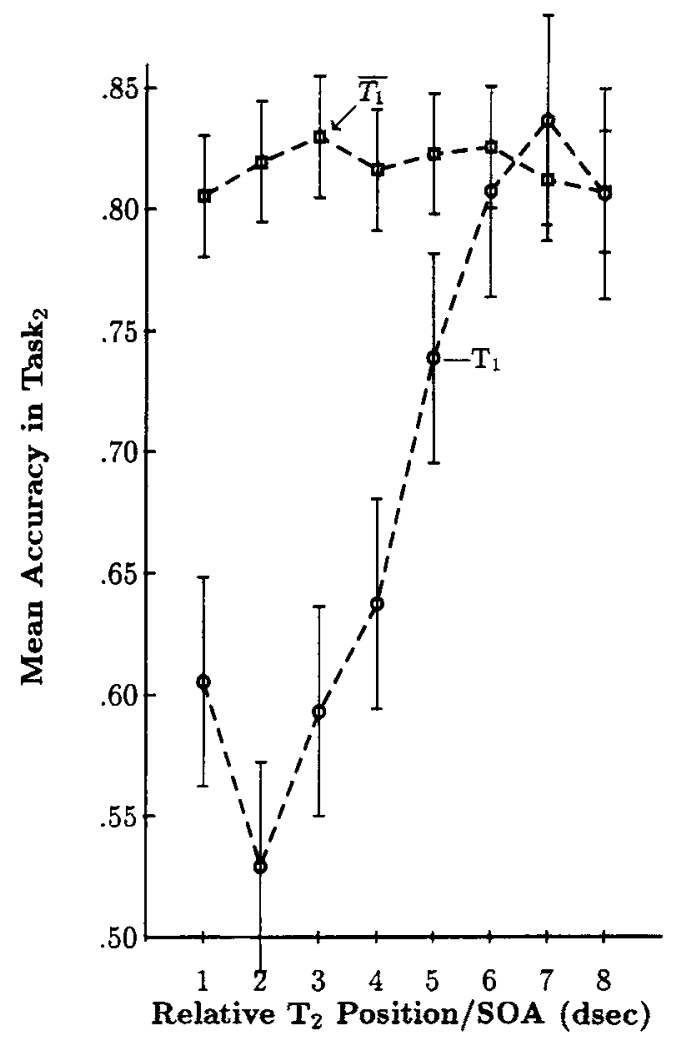

Figure 3. Results from Experiment 2. Mean proportion correct discrimination of $T_{2}(X$ vs. $Y)$ in $T_{1}$-absent trials (control trials, labeled $\bar{T}_{1}$ ) versus $T_{1}$-present trials (experimental trials, labeled $\left.T_{1}\right) .1$ decisecond (dsec) $=100$ msec. The error bars are $95 \%$ within-subjects confidence intervals appropriate for comparisons of means across SOAs within each function $\left(T_{1}\right.$ or $\left.\overline{T_{1}}\right)$.
$A B_{5.8}$ score $\left[.020 \pm .035 ; F(1,18)=76.23, M S_{\mathrm{e}}=\right.$ $0.005273, p<.0001]$.

Performance in Task ${ }_{1}$, for the $T_{1}$-present trials, was very good, with a mean of $93.7 \%$ correct. Accuracy in Task did not depend on the SOA at which the $\mathrm{X}$ or $\mathrm{Y}$ was presented $\left[F(7,126)=1.06, M S_{\mathrm{e}}=0.0016, p>.39\right]$

The mean RT in Task 1 was $530 \pm 8 \mathrm{msec}$ and did not differ appreciably across SOA $\left[F(7,126)=1.47, M S_{\mathrm{e}}=\right.$ $310.2, p>.18]$. These results suggest that the occurrence of $T_{2}$ did not significantly influence the processing of $T_{1}$.

As in Experiment 1, a large $\mathrm{AB}$ was obtained with a $2 \mathrm{AD}$ between $\mathrm{X}$ and $\mathrm{Y}$ in Task 2 .

\section{COMBINED ANALYSIS OF EXPERIMENTS 1 AND 2}

The means for Experiments 1 and 2 are shown in Figure 4 . The error bars are $95 \%$ confidence intervals computed from separate ANOVAs, one for each SOA, in which the speeded versus unspeeded factor was a betweensubjects variable. The interaction between $A B_{1,4}$ versus $A B_{5,8}$ scores with experiments $\left[F(1,36)=10.01, M S_{\mathrm{e}}=\right.$ $0.004124, p<.0035]$ confirms what is evident in Fig ure 4: There was a larger $A B$ effect in the speeded condition than in the unspeeded condition. This interpretation was corroborated further by a direct comparison of the $A B_{1,4}$ scores across the unspeeded $(.167 \pm .026)$ versus the speeded $(.226 \pm .035)$ condition $\left[F(1,36)=7.95 M S_{\mathrm{e}}=\right.$ $0.004110, p<.008]$.

\section{EXPERIMENT 3

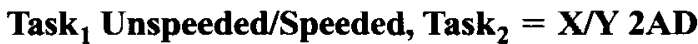

The results of Experiments 1-2 produced interesting evidence concerning the effects of requiring a speeded response in Task . This experiment provided additional evidence, using a within-subjects design. A within-subjects

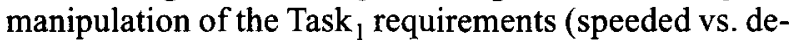
layed response) provided a replication of the effects found in the previous experiments and showed that these effects can be made to come and go, from block to block, depending on the instructions (and presumably on how the subjects scheduled the operations required to perform Task ${ }_{1}$ ).

This experiment was like Experiments 1 and 2, except that each subject performed both unspeeded and speeded versions of Task ${ }_{1}$, in different blocks of trials. Each subject performed 640 trials, 320 with an unspeeded Task

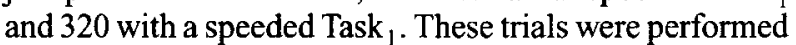
in blocks of 64 , with the speeded/unspeeded variable alternating from block to block. Half of the subjects started with Task unspeeded; the other half started with Task $_{1}$ speeded. The experiment began with 2 practice blocks of 32 trials, one speeded and one unspeeded.

\section{Results and Discussion}

Task $_{2}$ performance is graphed in Figure 5. The error bars for $T_{1}$-present trials (except at 400 -msec SOA; see 


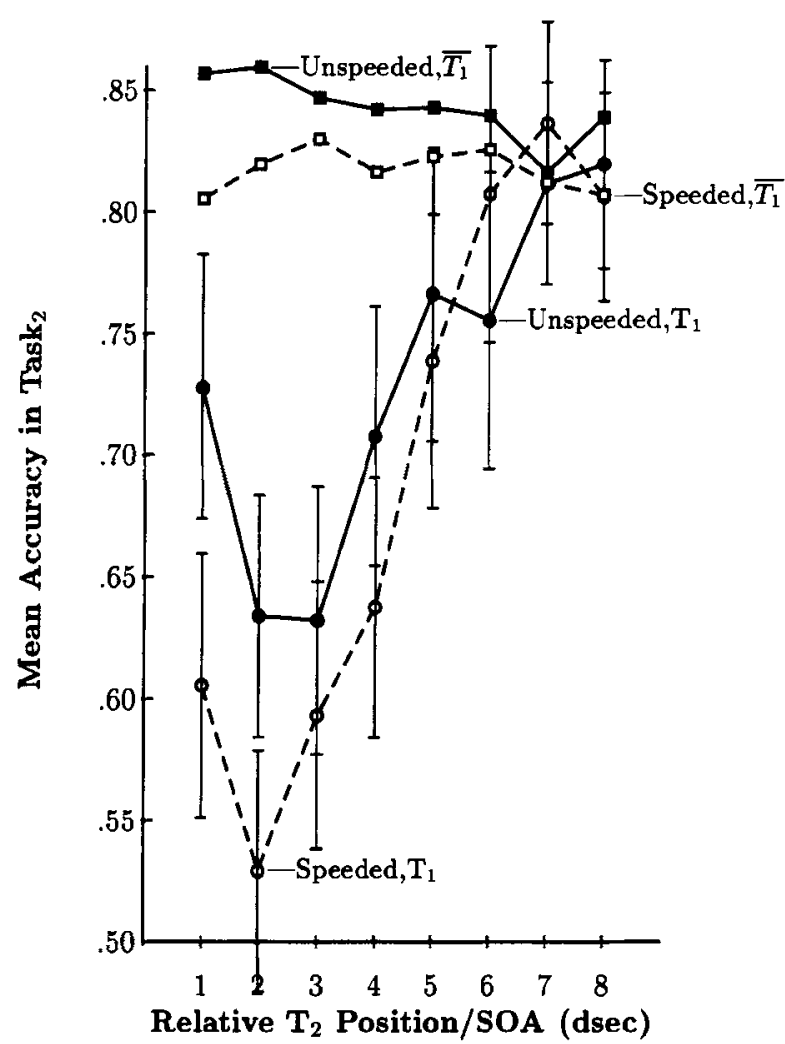

Figure 4. Results from Experiments 1 and 2. Mean proportion correct discrimination of $T_{2}\left(X\right.$ vs. $Y$ ) in $T_{1}$-absent trials (control trials, labeled $\bar{T}_{1}$ ) versus $T_{1}$-present trials (experimental trials, labeled $T_{1}$ ). Circles are for $T_{1}$-present trials; squares are for $T_{1}$ absent $\left(\overline{T_{1}}\right)$ trials. Solid lines and filled symbols are for unspeeded Task $_{1}$ conditions (Experiment 1); dashed lines and unfilled symbols are for speeded Task ${ }_{1}$ conditions (Experiment 2). 1 decisecond (dsec) $=100 \mathrm{msec}$. The error bars are $95 \%$ between-subjects confidence intervals appropriate for comparing the means in $T_{1^{-}}$ present trials at a given SOA.

below) show $95 \%$ within-subjects confidence intervals estimated from separate ANOVAs at each SOA in which only the results from $T_{1}$-present trials were considered. Clearly, the means for $\mathrm{T}_{1}$-present trials at 400 -msec SOA did not differ. The error bars for this SOA are the $95 \%$ within-subjects confidence interval appropriate for estimating the effects of SOA for $T_{1}$-present trials (estimated from a separate ANOVA in which results across speeded and unspeeded trials were pooled). As in Experiments $1-2$, there was a robust $A B$ effect in Task $_{2}$, which required a $2 A D$ between $X$ and $Y$. The error bars shown at the 200-msec SOA for the $T_{1}$-absent condition show the within-subjects $95 \%$ confidence interval appropriate for comparisons among the means for $\mathrm{T}_{1}$-absent trials.

The mean $A B_{1,4}$ score was .144 \pm .023 in the unspeeded condition and .187 \pm .023 in the speeded condition, whereas the $A B_{5,8}$ score was $.055 \pm .023$ in the unspeeded condition and $.006 \pm .023$ in the speeded condition, resulting in a significant interaction indicating a larger $\mathrm{AB}$ effect in the speeded condition than in the unspeeded con- dition $\left[F(1,21)=16.93, M S_{\mathrm{e}}=0.002725, p<.0005\right]$. A separate ANOVA comparing just the $A B_{1,4}$ scores confirmed that the deficit in Task ${ }_{2}$ performance was greater for the speeded condition than for the unspeeded condition $\left[F(1,21)=5.56, M S_{\mathrm{e}}=0.003675, p<.03\right]$.

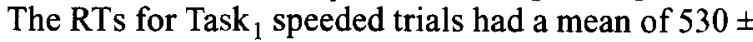
$13 \mathrm{msec}$ that did not vary significantly across $\mathrm{SOA}(F<1)$, suggesting that the time at which $T_{2}$ was presented did not strongly affect the processing of $\mathrm{T}_{1}$. The proportion of correct responses in Task ${ }_{1}$ was .975 for the unspeeded condition and .950 for the speeded condition $[F(1,21)=$ $\left.16.45, M S_{\mathrm{e}}=0.003267, p<.0006\right]$. Accuracy did not vary significantly with SOA $\left[F(7,147)=1.75, M S_{\mathrm{e}}=\right.$ $0.001993, p>.10]$, and the interaction between SOA and Task $_{1}$ condition was also not significant $[F(7,147)=1.10$, $\left.M S_{\mathrm{e}}=0.001440, p>.36\right]$.

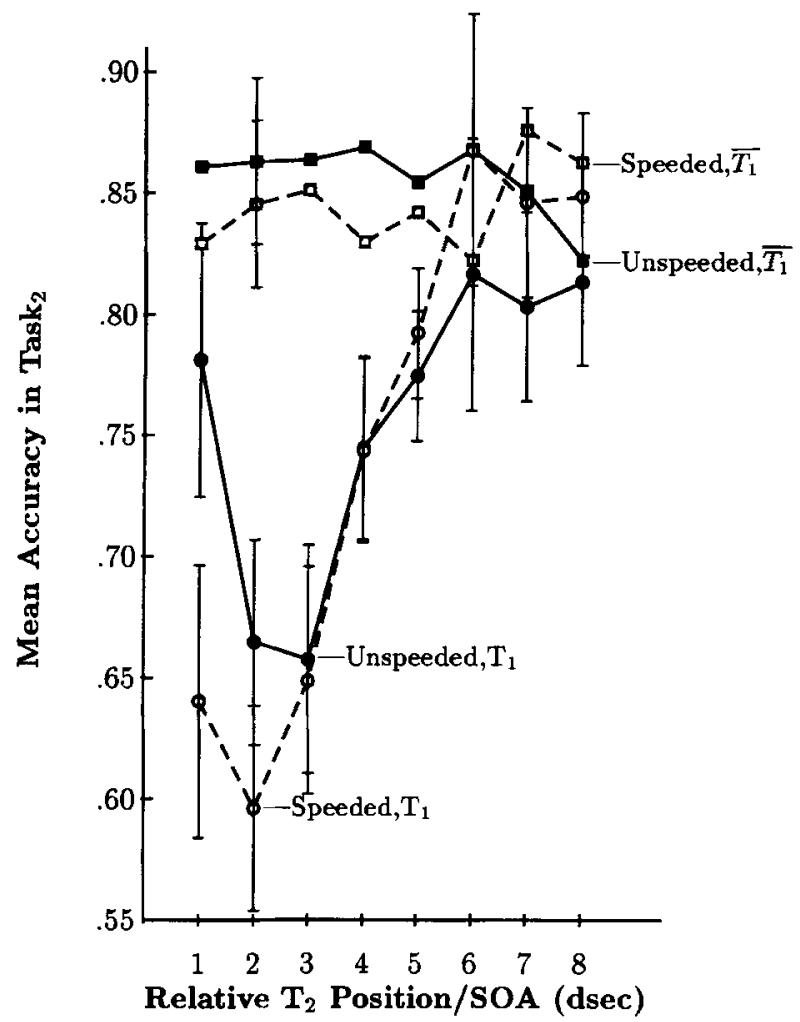

Figure 5. Results from Experiment 3. Mean proportion correct discrimination of $T_{2}\left(X\right.$ vs. $Y$ ) in $T_{1}$-absent (control) versus $T_{1}$ present (experimental) trials for unspeeded versus speeded Task conditions. $\bar{T}_{1}$ designates $T_{1}$-absent trials. $T_{1}$ designates $T_{1}$-present trials. Circles are for $T_{1}$-present trials; squares are for $T_{1}$-absent $\left(\overline{T_{1}}\right)$ trials. Solid lines and filled symbols are for unspeeded Task conditions; dashed lines and unfilled symbols are for speeded

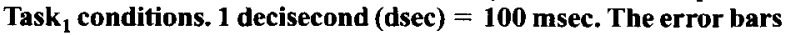
are $95 \%$ within-subjects confidence intervals appropriate for comparing the means in $T_{1}$-present trials at a given SOA. The error bars for $T_{1}$-present trials at the 4-dsec SOA are appropriate for comparisons across SOAs of the mean of speeded and unspeeded trials in $T_{1}$-present. The error bars at the 2-dsec SOA for $T_{1}$-absent trials are appropriate for comparisons across SOAs within $T_{1}$-absent conditions. 


\section{COMBINED ANALYSES OF EXPERIMENTS 1-3}

In order to maximize the precision of the estimates of condition means and thus maximize statistical power to corroborate the effects that appeared in the pattern of means when comparing performance across unspeeded

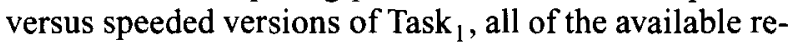
sults from Experiments 1-3 were combined into a single analysis. The results from Experiments 1 and 2 were shown in Figure 4, and those for Experiment 3 in Figure 5. In order to facilitate the analysis, the results from Experiment 3 in the combined ANOVA were treated as an independent group design. (Although this method of analysis reduced power to detect effects for those subjects, it made it easier to combine the results with those from the first two experiments.) The resulting analysis treated speeded versus unspeeded Task ${ }_{1}$ requirements as a between-subjects factor and $\mathrm{T}_{1}$-absent/present and SOA as within-subjects factors. The means are shown in Fig-

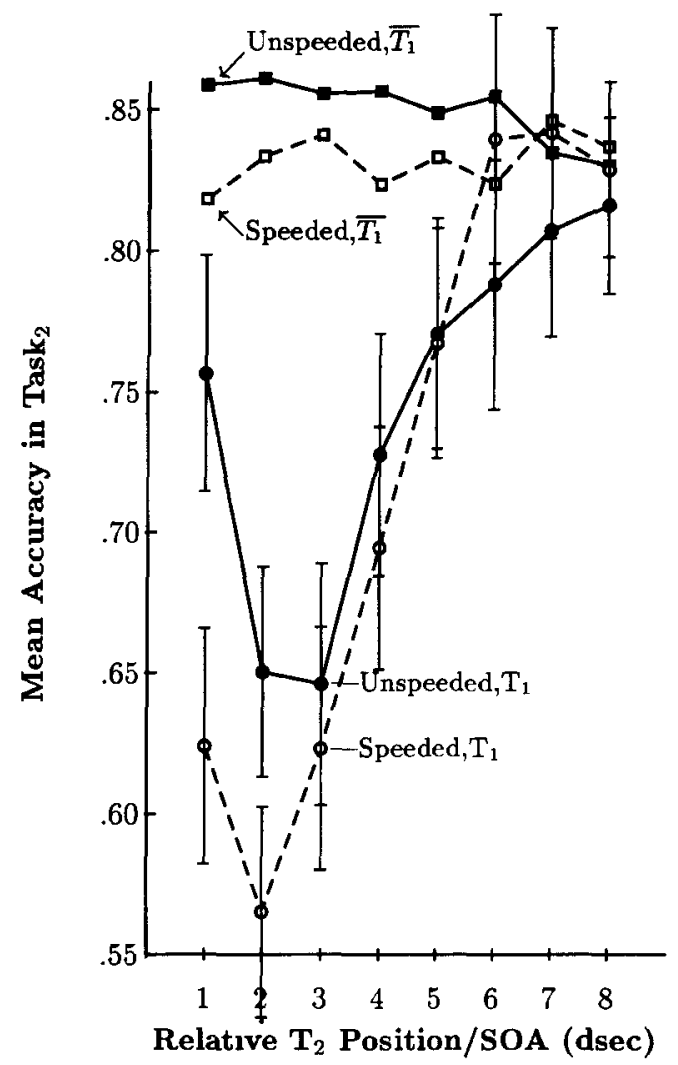

Figure 6. Results from Experiments 1-3 combined. Mean proportion correct performance in Task in $\mathrm{T}_{1}$-absent (control) versus $T_{1}$-present (experimental) trials for unspeeded versus speeded Task ${ }_{1}$ conditions. $\overline{T_{1}}$ designates $T_{1}$-absent trials. $T_{1}$ designates $T_{1}$ present trials. Circles are for $T_{1}$-present trials; squares are for $T_{1}$-absent $\left(\overline{T_{1}}\right)$ trials. Solid lines and filled symbols are for unspeeded Task ${ }_{1}$ conditions; dashed lines and unfilled symbols are

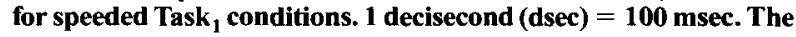
error bars are $95 \%$ within-subjects confidence intervals appropriate for comparing the means in $\mathrm{T}_{1}$-present trials at a given SOA. ure 6 . The error bars are $95 \%$ between-subjects confidence intervals estimated for $T_{1}$-present trials in a separate ANOVA for each SOA.

For unspeeded Task 1 trials, the $A B_{1,4}$ score was $.155 \pm$ .023 and the $A B_{5,8}$ score was $.055 \pm .023$; for speeded Task ${ }_{1}$ trials, the $A B_{1,4}$ Score was $.205 \pm .023$ and the $A B_{5,8}$ score was $.013 \pm .023$. This pattern of means produced a significant two-way interaction between the unspeeded versus speeded factor and the $\mathrm{AB}$ scores factor [short vs. long SOAs; $F(1,80)=16.55, M S_{\mathrm{e}}=0.005282, p<$ $.0001]$. As can be inferred from the confidence intervals, the $A B_{1,4}$ scores differed across the speeded versus unspeeded groups-confirmed in a separate ANOVA in which just these scores were analyzed in a between-subjects analysis $\left[F(1,80)=8.21, M S_{\mathrm{e}}=0.006319, p<.006\right]$.

As can be seen in Figure 6, the results for $T_{1}$-present trials in the speeded condition converged with those of $\mathrm{T}_{1}$-absent trials at the longer SOAs. In contrast, those for the unspeeded condition appeared to approach an asymptote that was below the control level for $T_{1}$-absent unspeeded trials. These impressions were corroborated by the following analyses. First, there was a significant difference between the mean $A B_{5,8}$ scores for the unspeeded versus the speeded condition $\left[F(1,80)=7.06, M S_{\mathrm{e}}=\right.$ $0.005131, p<.01$ ]; second, the mean $A B_{5,8}$ score for the unspeeded condition was significantly different from zero $\left[F(1,40)=25.55, M S_{\mathrm{e}}=0.0048, p<.0001\right]$; whereas third, the mean $A B_{5,8}$ score for the speeded condition was not significantly different from zero $[F(1,40)=1.19$, $\left.M S_{\mathrm{e}}=0.0055, p>.28\right]$.

These results are discussed further in later discussion sections. For the moment, it is sufficient to note that requiring a speeded response appears to cause differences in the results in Task ${ }_{2}$, both at shorter SOAs and at longer SOAs, relative to the results obtained in the delayedresponse paradigm.

\section{DISCUSSION OF THE EFFECTS OF TASK 1 RESPONSE REQUIREMENTS}

The new empirical discoveries described in Experiments 1-3 have been made possible by comparing the results from a delayed-response Task ${ }_{1}$ paradigm with those from an otherwise identical paradigm, except that a speeded response was required in Task ${ }_{1}$. There were two principal findings. First, the $\mathrm{AB}$ effect was larger at short SOAs when Task ${ }_{1}$ involved a speeded on-line response than when Task, involved a delayed response. Second, the results from $T_{1}$-present trials produced a crossover interaction, with better performance in the speeded condition than in the unspeeded condition at longer SOAs. Each of these results is discussed below.

For $\mathrm{T}_{1}$-absent trials, the effects of requiring a speeded response to $T_{1}$ (when it occurred) were minor. The means suggest that there might be a small additional cost in the speeded condition relative to the unspeeded condition, but, in separate statistical analyses of the combined results, the difference was not significant. The possibility of a small 
cost is mentioned here only because a difference was found (although not significant) in the comparison of Experiments 1 and 2 and within Experiment 3 . The main conclusion that can be drawn from these results is that, although there may have been some differential task preparation across the speeded versus unspeeded conditions, these differences were small and had only modest effects on the results. Thus, it seems reasonably safe to focus on the pattern of results obtained from trials in which $T_{1}$ was presented.

The results from $T_{1}$-present trials show that the $A B$ ef-

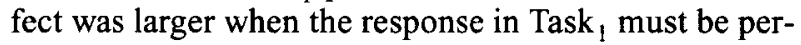
formed immediately, or on line. A smaller $\mathrm{AB}$ effect was observed when the response in Task 1 was made at the end of the trial, off line, without speed pressure. As can be seen in Figure 6, performance in Task ${ }_{2}$ was lower in the speeded condition than in the delayed condition at the shorter SOAs, but the opposite was found at longer SOAs. It is likely that the extra cost at the short SOAs caused by requiring an immediate response was due to the overlap between response selection and operations required to perform Task ${ }_{2}$. It is likely that this overlap is not present when the subject can make a delayed response. The results thus suggest that relatively late stages of processing, such as response selection, can modulate the magnitude of the $\mathrm{AB}$ phenomenon. A model based on the central interference theory designed to explain these findings is described in the General Discussion section.

Why should the $T_{1}$-present functions for unspeeded versus speeded Task ${ }_{1}$ responses cross over as SOA was increased? The account for this effect may be quite straightforward: in the delayed-response condition, a memory representation of $\mathrm{T}_{1}$ must be maintained in STM throughout the trial in order to make an accurate response at the end of the trial. It is known that memory load can influence the general efficiency of information processing (Logan, 1978). Furthermore, in both the two-stage model and the central interference theory, it is hypothesized that the primary locus of the $\mathrm{AB}$ effect is in the processes that consolidate information into STM. Thus, one might expect that memory load would influence performance (Scarborough, 1972). In contrast, in the speeded condition, once the response is selected, there is no further need to maintain an active representation of $T_{1}$ in STM. The subject can focus exclusively on Task ${ }_{2}$. Removing the need to maintain an active representation of $T_{1}$ in STM could reduce the central interference that may cause the $A B$ ef-

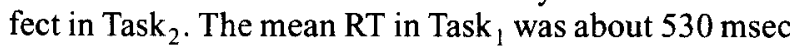
(Experiments 2 and 3), which is close to the point at which the $\mathrm{AB}$ functions cross over; this may not be coincidental. The difference in the on-line response selection processes at short SOAs and in the memory requirements at longer SOAs is thus consistent with the observed crossover pattern of results as SOA was increased from 100 to $800 \mathrm{msec}$.

\section{DEPENDENCY OF THE AB ON RT}

A hallmark of results consistent with postponement models of PRP effects is that certain aspects of perfor- mance in Task ${ }_{2}$ should be systematically related to the speed of responses in Task ${ }_{1}$. In the present paradigm, the $\mathrm{RT}$ in Task $\mathrm{R}_{1}\left(\mathrm{RT}_{1}\right)$ should reflect the time to perform several key cognitive operations required to perform Task . $_{1}$ Two critical processes should contribute significantly to the variance in $\mathrm{RT}_{1}$ : stimulus classification and response selection (Pashler, 1994). Thus, on trials in which $\mathrm{RT}_{1}$ was long, there is a higher probability that stimulus classification and response selection operations were slower than usual. Conversely, on trials in which $\mathrm{RT}_{1}$ was short, chances are that stimulus classification and response selection operations were faster than usual. Thus, when $\mathrm{RT}_{1}$ was short, the central operations that occupy the hypothetical central bottleneck likely did so for less time than when $\mathrm{RT}_{1}$ was long. If bottleneck processes are involved in the $\mathrm{AB}$ phenomenon, one would expect a smaller $\mathrm{AB}$ for fast responses to $T_{1}$ than for slow responses.

To examine the prediction that the magnitude of the AB effect would depend on speed of response in Task performance in Task ${ }_{2}$ was conditionalized on $\mathrm{RT}_{1}$. The general method of analysis was as follows. First, $T_{1}$ present trials (in which Task ${ }_{1}$ was speeded) were sorted by SOA separately for each subject. The RTs for correct responses in each cell were first screened for outliers, using a procedure that is a slight modification of the one described by Van Selst and Jolicoeur (1994b; the modification involves temporarily excluding the most extreme observation rather than the largest observation). ${ }^{1}$ No more than $2.5 \%$ of the correct trials were rejected, using this procedure. Note that removing outliers is likely to attenuate any Task ${ }_{2}$ dependency on $\mathrm{RT}_{1}$, since at least some of the long $\mathrm{RT}_{1} \mathrm{~s}$ so excluded were probably associated with poor performance in Task ${ }_{2}$ (as will become clear below).

The trials in each SOA category for each subject were then split into two categories, short versus long, by doing a median split on the $\mathrm{RT}_{1} \mathrm{~S}$ within each cell. Performance in Task ${ }_{2}$ was then computed separately at each SOA (and subject) within each category. In Experiment 2, this resulted in about 20 trials per SOA per subject, whereas the number was about 10 in Experiment 3. A finer split of the results was also performed by dividing the $\mathrm{RT}_{1} \mathrm{~s}$ into quartiles (within each cell for each subject) and aggregating the results across Experiments 2 and 3.

The results for Experiment 2 are shown in Figure 7. The error bars show 95\% within-subjects confidence intervals, appropriate for comparisons among the displayed means (estimated from an ANOVA in which the 16 means were treated as a single within-subjects factor). The interaction between SOA and short/long-RT ${ }_{1} \mathrm{~s}$ was significant $\left[F(7,126)=2.35, M S_{\mathrm{e}}=0.0068, p<\right.$ .028]. The mean for short $\mathrm{RT}_{1} \mathrm{~s}(.72)$ was higher than for long $\mathrm{RT}_{1} \mathrm{~s}\left[.67 ; F(1,18)=M S_{\mathrm{e}}=0.0095, p<.0001\right]$. Two aspects of the results are apparent in the figure. First, accuracy in Task ${ }_{2}$ at shorter SOAs was lower when $\mathrm{RT}_{1}$ was longer than when $\mathrm{RT}_{1}$ was shorter (except at an SOA of $200 \mathrm{msec}$, where accuracy was equivalent across short and long $\mathrm{RT}_{1} \mathrm{~s}$ ). This difference was similar at intermediate SOAs, with some convergence between the two 


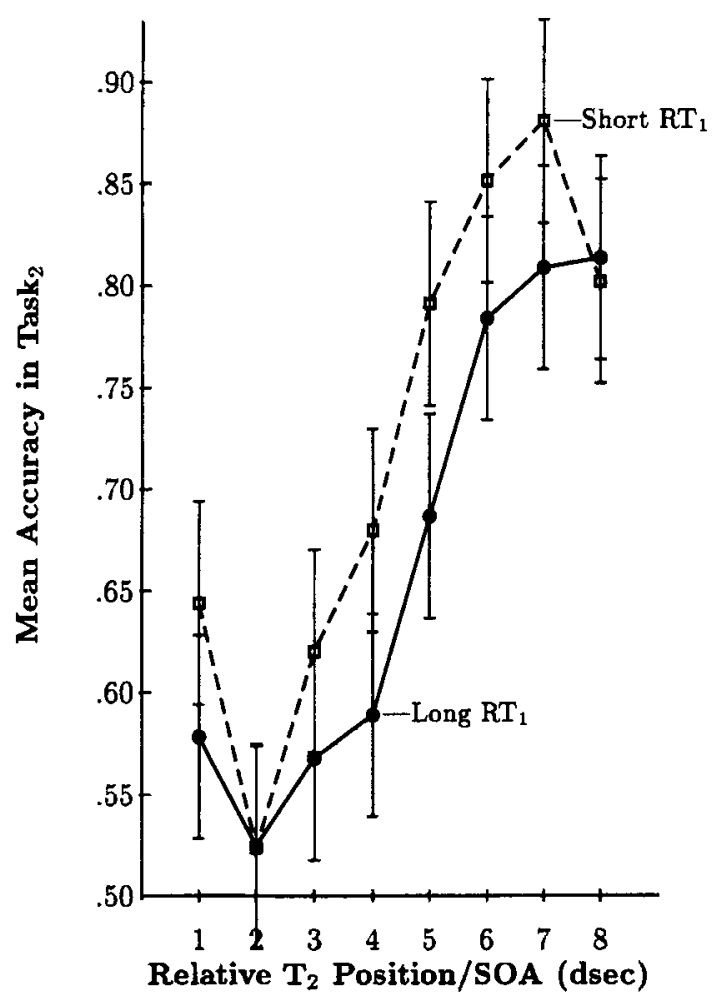

Figure 7. Results from Experiment 2. Mean proportion correct discrimination of $T_{2}(X$ vs. $Y$ ) depending on response times $(R T s)$ in Task ${ }_{1}$ (based on a median split of $T_{1}$-present trials only, following outlier screening). Unfilled squares and dashed lines are for short $\mathrm{RT}_{1} \mathrm{~s}$; filled circles and solid lines are for long $\mathrm{RT}_{1} \mathrm{~s}$. The error bars are $95 \%$ within-subjects confidence intervals appropriate for comparisons of the means in the figure. 1 decisecond (dsec) $=100 \mathrm{msec}$.

functions at the longest SOA. The lower accuracy in Task for longer $\mathrm{RT}_{1} \mathrm{~s}$ than for shorter $\mathrm{RT}_{1} \mathrm{~s}$ at almost every $\mathrm{SOA}$ suggests that a larger $A B$ effect was associated with longer $\mathrm{RT}_{1} \mathrm{~s}$. Second, given that performance in Task ${ }_{2}$ appeared to recover to near-baseline levels only at the longest SOA, the results also suggest that the $\mathrm{AB}$ effect had a longer duration when $\mathrm{RT}_{\text {, was }}$ longer.

The mean $\mathrm{RT}_{1}$ was $456 \mathrm{msec}$ for RTs shorter than the median and $606 \mathrm{msec}$ for RTs longer than the median. RTs did not vary significantly with $\operatorname{SOA}[F(7,126)=1.94$, $\left.M S_{\mathrm{e}}=636.104, p>.06\right]$, and the pattern of $\mathrm{RT}_{1} \mathrm{~s}$ for faster versus slower trials did not interact with $\operatorname{SOA}[F(7,126)=$ $\left.1.47, M S_{\mathrm{e}}=373.708, p>.18\right]$

The means showing the dependence of $\mathrm{Task}_{2}$ performance on $\mathrm{RT}_{1}$ for Experiment 3 are in Figure 8. Task 2 performance was better when the response to $T_{1}$ was fast $(.78)$ than when it was slow $\left[.72 ; F(1,21)=19.70, M S_{\mathrm{e}}=\right.$ $0.0150, p<.0003]$. The interaction between short/long$\mathrm{RT}_{1}$ and SOA was not significant, however $[F(7,147)=$ $\left.1.42, M S_{\mathrm{e}}=0.0178, p>.20\right]$.

The mean RT - was $451 \mathrm{msec}$ for RTs shorter than the median and $608 \mathrm{msec}$ for RTs longer than the median. RTs did not vary significantly with SOA $(F<1)$, and the pat- tern of $\mathrm{RT}_{1} \mathrm{~s}$ for faster versus slower trials did not interact with SOA $(F<1)$.

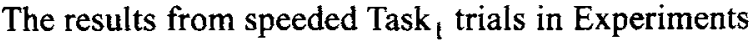
2 and 3 were also combined into a single analysis. Given the larger number of subjects contributing to the analysis, the trials were split on the basis of $\mathrm{RT}_{1}$ quartiles. The resulting means are in Figure 9. The error bars are 95\% within-subjects confidence intervals estimated from the error term in an ANOVA in which the SOA $\times$ quartile interaction was treated as a single factor with 32 levels (Loftus \& Masson, 1994). Error bars are plotted only for the means for the first (shortest $\mathrm{RT}_{1} \mathrm{~s}$ ) and fourth quartiles to reduce clutter. Task 2 performance was .77 for the first quartile, .74 for the second quartile, .73 for the third quartile, and .67 for the fourth quartile [averaging across all SOAs; $F(3,120)=20.85, M S_{\mathrm{e}}=0.025977, p<$ $.0001]$. The interaction between quartiles and SOA was also significant $\left[F(21,840)=2.12, M S_{\mathrm{e}}=0.025682\right.$, $p<.0025]$. The rank ordering of the means from the first to the fourth quartile was reasonably well preserved at most SOAs, except for the longest ones, where, as ex-

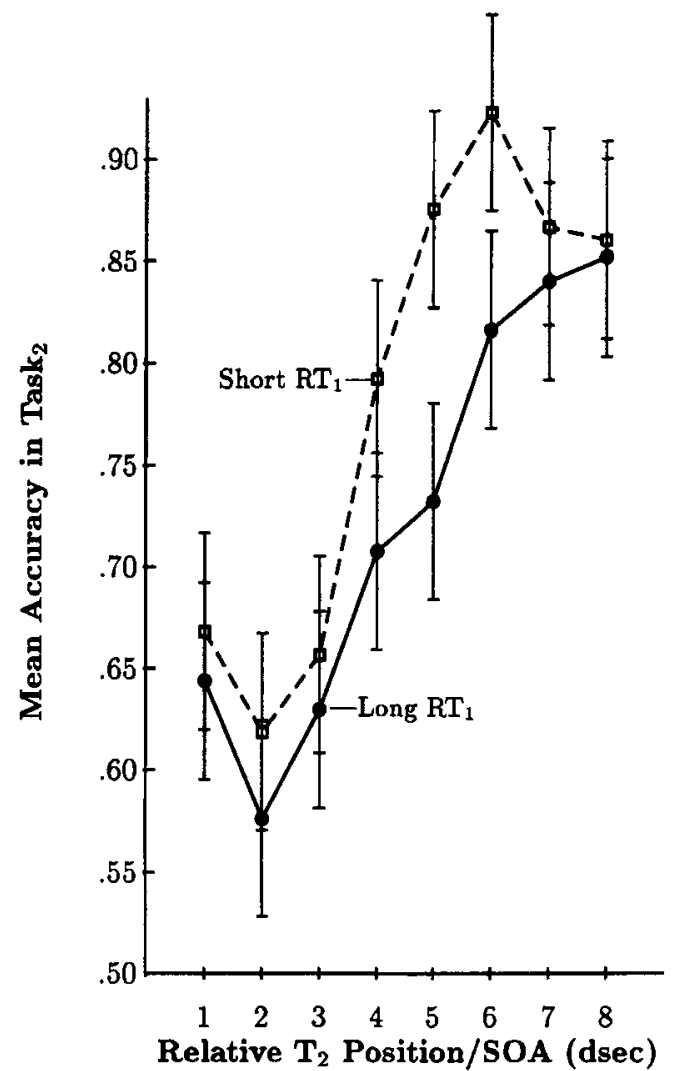

Figure 8. Results from Experiment 3. Mean proportion correct discrimination of $T_{2}(X v s . Y)$ depending on response times (RTs) in Task ${ }_{1}$ (based on a median split of $T_{1}$-present trials only, following outlier screening). Unfilled squares and dashed lines are for short $\mathrm{RT}_{1} \mathrm{~s}$; filled circles and solid lines are for long $\mathrm{RT}_{1} \mathrm{~s}$. The error bars are $95 \%$ within-subjects confidence intervals appropriate for comparisons of the means in the figure. 1 decisecond (dsec) $=100$ msec 


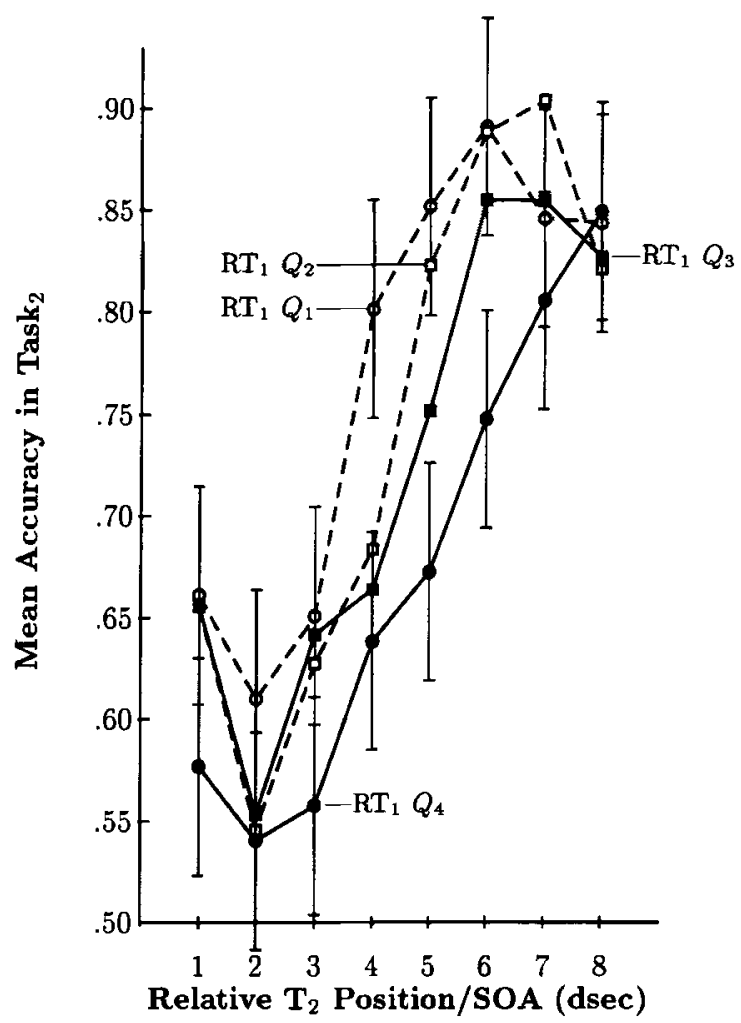

Figure 9. Results from Experiments 2-3. Mean proportion correct discrimination of $T_{2}(X$ vs. $Y)$ depending on response times (RTs) in Task (based on a quartile split of $T_{1}$-present trials only, following outlier screening). $Q_{1}$ designates means for the first quartile (fastest responses), $Q_{2}$ the second quartile, $Q_{3}$ the third quartile, and $\mathrm{Q}_{4}$ the fourth quartile (slowest responses). To facilitate interpretation, the $Q_{1}$ function is plotted with unfilled circles joined by dashed lines; the $Q_{2}$ function is plotted with unfilled squares joined by dashed lines; the $Q_{3}$ function is plotted with filled squares joined by solid lines; and the $Q_{4}$ function is plotted with filled circles joined by solid lines. The error bars are $95 \%$ within-subjects confidence intervals appropriate for comparisons of the means in the figure. 1 decisecond (dsec) = 100 msec.

pected, the functions converged. This convergence was expected because the response in Task ${ }_{1}\left(\mathrm{R}_{1}\right)$ was already executed on most trials by the time $T_{2}$ was shown at the longest $\mathrm{SOA}$ (mean $\mathrm{RT}_{1}=416 \mathrm{msec}$ for the first quartile, $490 \mathrm{msec}$ for the second quartile, $552 \mathrm{msec}$ for the third quartile, and $664 \mathrm{msec}$ for the fourth quartile). Central involvement required to process $T_{1}$ should usually be over for the longer SOAs. Thus, on the view that the association between $\mathrm{RT}_{1}$ and $\mathrm{AB}$ magnitude is mediated by differential durations of central involvement required to perform Task $_{1}$, there is no reason to expect $\mathrm{RT}_{1}$ variations to continue to affect performance in Task ${ }_{2}$ long after $R_{1}$.

As can be seen in Figures 7-9, performance in Task showed a strong dependence on the speed of the response in Task ${ }_{1}$ within both experiments and in the combined analysis. The analyses were based on a within-SOA within- subjects split of the RTs in Task ${ }_{1}$. The results show a micro dependence (De Jong \& Sweet, 1994) between the trial-totrial processing speed in Task 1 and performance in Task ${ }_{2}$.

\section{GENERAL DISCUSSION}

The $\mathrm{AB}$ phenomenon is interesting because it is likely that it reveals a fundamental limitation in our ability to process perceptual input. Performance in Task ${ }_{2}$ suffers for a period of time when $T_{2}$ is presented shortly after $T_{1}$, but only when $T_{1}$ is actively processed by the observer. The experiments reported in the foregoing sections have revealed several new properties of the $A B$ phenomenon that can be used to advance our theoretical understanding of this phenomenon and of the human information processing system in general. These new properties can be summarized as follows. First, the shape of the AB function ( $T_{1}$-present trials) changes in a characteristic way when the response in Task ${ }_{1}$ is changed from off line to on line. Accuracy in Task ${ }_{2}$ was worse at short SOAs when $R_{1}$ was performed on-line than off-line, whereas the opposite was true at longer SOAs. The second observation, made possible by requiring a speeded $\mathrm{R}_{1}$ and measuring $\mathrm{RT}_{1}$, was that the magnitude of the $A B$ effect was larger for longer $\mathrm{RT}_{1} \mathrm{~s}$ than for shorter $\mathrm{RT}_{1} \mathrm{~s}$ (see Figures 7-9).

\section{Task $_{1}$ Response Requirements}

It is likely that the effects of requiring an immediate versus a delayed response in Task ${ }_{1}$ occur at a relatively late stage of processing (i.e., at a postperceptual stage). It is likely that response selection (a postperceptual stage of processing) was engaged during the display of the RSVP stream in the speeded-response condition but that response selection was delayed until the end of the trial in the delayed-response condition. Other evidence (Jolicoeur, in press-a, in press-b) also suggests that manipulations of the duration of response-level stages of processing, most likely response selection, can have significant effects on the shape and duration of the $A B$ effect. The following paragraphs contain a discussion of the finding that the magnitude of the $A B$ effect was modulated by the response requirements (speeded vs. delayed) in Task ${ }_{1}$ in the context of the models presented in the introduction.

None of the models, other than the central interference theory, predicted that requiring an immediate response in Task ${ }_{1}$ would have an effect on the magnitude, duration, or shape of the AB function. Ward et al. (1996) were the clearest on this point in that their attentional dwell model explicitly excludes late processes, such as those associated with response selection, as being causally involved in the $\mathrm{AB}$ phenomenon. In the attentional dwell model, the locus of the AB effect is in the capacity-limited process of transferring representations from Level 1 to Level 2, which occurs before additional processing, such as response selection, can take place.

Although the authors of the other models did not explicitly exclude response selection as being causal in the 
AB phenomenon, the models have no natural way to account for effects at this stage of processing. In the attentional gate model (Raymond et al., 1992), the AB is caused by the closing of an attentional filter designed to protect pattern recognition mechanisms from posttarget patterned noise. The opening and closing of the gate were postulated to depend on the characteristics of perceptual stimulation (e.g., whether $T_{1}+1$ in an RSVP stream was a patterned stimulus or a blank interval). There is no mechanism in the model that can allow processing that takes place after $T_{1}$ has been identified (e.g., response selection) to influence the opening and closing of the attentional gate or to affect the efficiency of the filter.

In the similarity theory (Shapiro et al., 1994), the AB effect is caused by limited resources in the assignment of weights to items that enter VSTM. Output from VSTM is then based on these weights. Presumably, responses are made to items only after they have been transferred out of VSTM and passed on to a later stage of processing that makes contact with other cognitive control mechanisms. According to the model, the causal mechanisms that produce the $\mathrm{AB}$ effect all take place before responserelated processes. Thus, according to this model, there was no reason to expect that requiring an immediate versus a delayed response should have had any effect on the magnitude or shape of the $\mathrm{AB}$ effect.

According to the two-stage model (Chun \& Potter, 1995), the locus of the AB effect is a capacity-limited process by which items are transferred from a fragile and temporary form of representation (Level 1 in Duncan, 1980) to a more permanent form of representation (Level 2) that can serve as the basis for overt responses and recall. As in the attentional dwell model, the manipulation of the

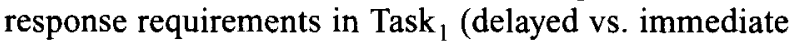
response) should have an effect at a stage of processing that occurs after the hypothesized locus of the $\mathrm{AB}$ effect. Therefore, this model also has no obvious way to deal with the effects of Task ${ }_{1}$ response requirements.

\section{Dependency of the $\mathrm{AB}$ on $\mathrm{RT}_{1}$}

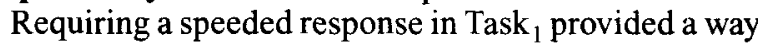
to estimate the duration of processing required to perform Task ${ }_{1}$ and to relate it to the magnitude of the AB effect observed in Task $\mathrm{T}_{2}$. A larger AB effect was found when $\mathrm{RT}_{1}$ was longer than when $\mathrm{RT}_{1}$ was shorter (see Figures 7-9). Such trial-to-trial dependencies have been used to argue against resource accounts of dual-task slowing in the PRP literature (Pashler, 1994). The argument has the following form: Suppose that there is a fixed pool of resources that can be divided for allocation to either Task or Task $_{2}$. On trials in which RT 1 was short, the inference would be that more of the available resources had been allocated to Task ${ }_{1}$ and, therefore, fewer were available for Task ${ }_{2}$. Conversely, if RT 1 was long, fewer resources were allocated to Task $_{1}$, leaving more for Task $\mathrm{K}_{2}$. In this view, therefore, short $\mathrm{RT}_{1} \mathrm{~s}$ should be associated with worse performance in Task ${ }_{2}$, and long $\mathrm{RT}_{1} \mathrm{~s}$ should be associated with better performance in Task in other words, exactly the opposite of what was found. Thus, the observed pattern of results is not consistent with this interpretation of resource sharing.

The similarity theory (Shapiro et al., 1994) and the attentional dwell model (Duncan et al., 1994; Ward et al., 1996) both incorporate aspects of resource models. In similarity theory, the notion of resources appears in how strongly items are represented in VSTM. Items in VSTM receive weights that depend on two factors: how well they match selection templates for $T_{1}$ and $T_{2}$ and the level of available resources. Resources must be shared by the items in VSTM, with larger weights assigned to the first items to enter VSTM because available resources have not been allocated to previous items. That is, the weights that items receive depend on a pool of resources that must be shared by all the items in VSTM. In the attentional dwell model, objects compete for passage from a first level of representation to a second level (Duncan, 1980), and it is assumed that resources devoted to one item are not available for others. If we suppose that the pool of available resources in these models is fixed and that items that receive more resources are processed faster, the models should predict that faster $\mathrm{RT}_{1} \mathrm{~s}$ should be associated with larger $\mathrm{AB}$ effects. The observed dependency between $\mathrm{RT}_{1}$ and the magnitude of the $A B$ is inconsistent with this prediction, however.

Another interpretation of the notion of resources could predict simple positive correlations between $\mathrm{RT}_{1}$ and $\mathrm{AB}$ magnitude. In this interpretation, we relax the assumption that the pool of resources is fixed. For example, suppose that there are momentary fluctuations in total available resources. Some trials would occur while the total available resources are low. On these trials, performance in both tasks would be poor-a longer $\mathrm{RT}_{1}$ in Task $_{1}$ and lower accuracy in Task ${ }_{2}$ would result. On other trials, the available resources might be high, resulting in good performance in both tasks. This line of argument leads to a difficulty, however. It is not clear why performance in $\mathrm{Task}_{2}$ would converge to a common level at longer SOAs (see, e.g., Figure 9). If we assume that the relationship observed between $\mathrm{RT}_{1}$ and $\mathrm{AB}$ magnitude is the result of fluctuating levels of total available resources, on trials initiated at a time when resources were scarce, performance in Task ${ }_{2}$ should remain lower at all SOAs. Therefore, although the notion of a fluctuating pool of available resources could explain a simple main effect relationship between the duration of $\mathrm{RT}_{1}$ and performance in Task 2 , it cannot easily account for the more complex interaction that was observed.

In the context of the similarity theory and of the attentional dwell model, one would also expect that accuracy in Task ${ }_{1}$ would be strongly related to $\mathrm{RT}_{1}$, if the relationship between $\mathrm{RT}_{1}$ and accuracy in Task $\mathrm{T}_{2}$ is to be explained by appeal to fluctuating resources. On trials with a smaller pool of resources, lower accuracy in Task and longer $\mathrm{RT}_{1} \mathrm{~s}$ should be found. 
To test this prediction, the trials for each subject and for each SOA were divided into four bins on the basis of $\mathrm{RT}_{1}$ quartiles. The mean accuracy in Task $_{1}$ was computed within each bin. The patterns of results were similar in Experiments 2 and 3 (interaction $F<1$ ), and so only the results from a combined analysis that considered speeded Task $_{1}$ trials from all 41 subjects are presented here. The mean accuracy in Task $_{1}$ was .915 for the first quartile (fast $\left.\mathrm{RT}_{1} \mathrm{~s}\right), .958$ for the second quartile, .951 for the third quartile, and .956 for the fourth quartile $[F(3,120)=$ $\left.10.90, M S_{\mathrm{e}}=0.012217, p<.0001\right]$. Accuracy in Task 1 did not depend on when $T_{2}$ was presented $[F(7,280)=$ $1.53, M S_{\mathrm{e}}=0.008093, p>.15$ ] or on the interaction between $\mathrm{RT}_{1}$ quartile and SOA $\left[F(21,840)=1.28, M S_{\mathrm{e}}=\right.$ $0.006876, p>.18]$.

The results were clearcut: Accuracy in Task ${ }_{1}$ was slightly lower when $\mathrm{RT}_{1}$ was short (first quartile) but did not vary as a function of $\mathrm{RT}_{1}$ for longer $\mathrm{RT}_{1} \mathrm{~s}$ (accuracy was constant across the remaining three quartiles). This pattern of results provides evidence against a resources account of the relationship between $\mathrm{RT}_{1}$ and accuracy in Task $_{1}$ and Task ${ }_{2}$. On that account, fewer resources should have produced longer $\mathrm{RT}_{1} \mathrm{~s}$ and worse accuracy in both tasks. Instead, the relationship between $\mathrm{RT}_{1}$ and accuracy in Task $_{1}$ was of one kind (worse performance at shorter $\mathrm{RT}_{1}$ than at longer $\mathrm{RT}_{1}$, and only for the first quartile), whereas it was altogether different in Task ${ }_{2}$ (accuracy decreased monotonically as $\mathrm{RT}_{1}$ increased). These considerations suggest that the results are not explained very well by resource models.

The relationship between $\mathrm{AB}$ magnitude and $\mathrm{RT}_{1}$ is also not easily accounted for by the attentional gate model (Raymond et al., 1992). There is simply no a priori way to relate the speed of processing of $T_{1}$ to the filtering efficiency of the gate or to the speed of the gate.

On the other hand, there is a way to relate the duration of processing devoted to $T_{1}$ to the magnitude of the $A B$ on the basis of the two-stage model (Chun \& Potter, 1995). All we need to suppose is that $\mathrm{RT}_{1}$ will depend, at least in part, on how long $T_{1}$ is processed through Stage 2 . The duration of Stage 2 processing for $T_{1}$ directly affects how long $T_{2}$ has to wait before it can be processed through Stage 2 and, therefore, how long the Stage 1 representation of $\mathrm{T}_{2}$ decays during the waiting period. A longer period of waiting leads to more decay. Thus, a longer $\mathrm{RT}_{1}$ should be associated with a longer period of Stage 2 processing for $\mathrm{T}_{1}$, which, in turn, should produce a larger $\mathrm{AB}$ effect in $\operatorname{Task}_{2}$, which is what was observed. The central interference theory makes the same prediction for the same fundamental reason, as outlined in the section entitled Central Interference Theory.

\section{One or More Loci?}

How well each model can account for the two main new results of the experiments can be summarized as follows:

\footnotetext{
Attentional Gate Model

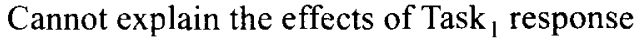
requirements
}

Cannot explain the relationship between $\mathrm{RT}_{1}$ and $\mathrm{AB}$ magnitude

Similarity Theory

Cannot explain the effects of Task ${ }_{1}$ response requirements

The VSTM resources component of the model predicts a different relationship between $\mathrm{RT}_{1}, \mathrm{AB}$ magnitude, and accuracy in Task ${ }_{1}$ than was observed

\section{Attentional Dwell Model}

Cannot explain the effects of Task 1 response requirements

Resources account of processing in Stage 2 predicts a different relationship between $R T_{1}, A B$ magnitude, and accuracy in Task ${ }_{1}$ than was observed

Two-stage Model

Cannot explain the effects of Task ${ }_{1}$ response requirements

Can explain the relationship between $\mathrm{RT}_{1}$ and $\mathrm{AB}$ magnitude

On this report card, the two-stage model fares the best, because it is the only one that can provide an account of the relationship between $\mathrm{RT}_{1}$ and $\mathrm{AB}$ magnitude without further elaboration or assumptions. None of the models has a good way to deal with effects of Task ${ }_{1}$ response requirements, because, in every case, response selection operations take place after the locus of dual-task interference postulated in the model. One way in which we could deal with the effects of Task ${ }_{1}$ response requirements is to hypothesize that there are multiple loci for the $\mathrm{AB}$ phenomenon. In this view, response selection effects would constitute a new phenomenon, a new attentional blink, which we might label $\mathrm{AB}^{\mathrm{RS}}$, to distinguish it from the previously discovered effect, $A B$. For the attentional gate model, we might label the effects of the attentional gate $A B^{G}$, and the effects observed in this article could be represented as $A B^{G} \oplus A B^{R S}$. For the two-stage model and the attentional dwell model, we might label the $A B$ effect produced by the transfer from Level 1 to Level 2 as $A B^{1 \rightarrow 2}$, and the effects observed in this article as $\mathrm{AB}^{1 \rightarrow 2} \oplus \mathrm{AB}^{\mathrm{RS}}$. Similarly, for the similarity theory, we might have a combined effect represented by ABVSTM $\oplus$ $\mathrm{AB}^{\mathrm{RS}}$. We could go as far as to suppose that there are more than two loci and that the observed $A B$ effect reflects some combination of all of the postulated loci proposed by various authors, which we could represent by

$$
A B \equiv A B^{\mathrm{G}} \oplus A B^{\mathrm{VSTM}} \oplus A B^{1 \rightarrow 2} \oplus A B^{\mathrm{RS}} .
$$

Other combinations involving $\mathrm{AB}^{\mathrm{RS}}$ are also possible. The present results cannot rule out any of these possibilities.

\section{Central Interference Theory}

The present results cannot refute models involving multiple loci. On the other hand, it may be possible to account for the results in a framework in which there is a single locus of interference in terms of the series of stages 
required to perform Task ${ }_{2}$. The purpose of this section is to present the outline of a theory in which there is only one fundamental locus of interaction between the two tasks.

The theory extends postponement models of the PRP effect to account for the AB phenomenon. The theory supposes that the critical stage of processing required to perform Task ${ }_{2}$ that is affected by concurrent processing in Task $_{1}$ is short-term consolidation (STC). STC is the process of encoding information into STM. The key concept in the theory is that certain cognitive processes require central mechanisms that are capacity limited. This capacity limitation imposes a seriality in the sequence of operations for certain combinations of operations but not for others. It is assumed that STC requires central capacity-limited processing. STC for Task ${ }_{2}$ can be delayed by a number of different operations that could be required for Task ${ }_{1}$.

Although response selection has been identified as one stage of processing that appears to require central limited-capacity processing (see, e.g., McCann \& Johnston, 1992; Pashler, 1994), it is not the only operation that can cause central PRP interference with other concurrent processing (see Pashler, 1994, for a recent review). There is good evidence that some aspects of mental rotation (Ruthruff, Miller, \& Lachmann, 1995; Van Selst \& Jolicoeur, 1994a), and retrieval from long-term memory (LTM; Carrier \& Pashler, 1995) also require central processing and cause PRP interference with other tasks.

The central interference theory incorporates the interpretation of PRP interference presented above. In this view, response selection is not the only stage capable of producing central interference; it is only one operation in a family of operations that can do so. In the context of the $\mathrm{AB}$ phenomenon, several cognitive operations can cause interference on the STC of $T_{2}$. The set of such operations includes response selection, the STC of $\mathrm{T}_{1}$ (as in the two-stage model), and mental rotation. It is also likely that central involvement required to switch between two tasks (Rogers \& Monsell, 1995) can also interfere with the STC of $\mathrm{T}_{2}$ (Potter, Chun, Banks, \& Muckenhoupt, in press), as might retrieval from LTM (Carrier \& Pashler, 1995). This conceptualization should make it clear that the two-stage model is a special case of the central interference theory. In the two-stage model, only Stage 2 processing of $T_{1}$ can postpone Stage 2 processing of $T_{2}$. In the central interference theory, the set of operations that can postpone $\mathrm{STC}_{2}$ includes $\mathrm{STC}_{1}$, but it also includes other operations, such as response selection, mental rotation, retrieval from LTM, and probably task switching (Potter et al., in press). In this framework, therefore, there is one key stage of processing required to perform Task ${ }_{2}$ that is subject to interference- the STC of $\mathrm{T}_{2}$ - and as such there is only one fundamental locus of interference in the sequence of processing required to

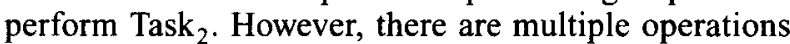
in Task ${ }_{1}$ that can interfere with the STC of $\mathrm{T}_{2}$. Thus, one could describe interference of $\mathrm{STC}_{2}$ by $\mathrm{STC}_{1}\left(\mathrm{STC}_{\mathrm{ST}}\right)$, or by $\mathrm{RS}_{1}$ (response selection in Task $_{1}$ ) as different causes of the $\mathrm{AB}$ effect, with both causes acting on a single stage of processing in Task . $_{2}$

Figure 10 illustrates a model of interactions between stages of processing required to perform the two tasks in the $A B$ paradigm that is based on the central interference theory. Figure $10 \mathrm{~A}$ shows these interactions for the delayedresponse paradigm. The model assumes that the earliest stages of encoding, called sensory encoding (SE) and perceptual encoding (PE), can take place without central involvement and essentially without mutual interference across tasks. The most important assumption of the model is that some central involvement is required to encode information into STM. This encoding process, STC, cannot occur when central mechanisms are occupied with the processing required for another concurrent task.

The top sequence of stages of each panel in Figure 10 shows the stages necessary to perform Task . When Task $_{1}$ involves a delayed response, the main activity that engages central mechanisms is the process of STC postulated to be necessary to encode $\mathrm{T}_{1}$ into STM. STM is required because information about $T_{1}$ must be maintained throughout the remaining presentation of the RSVP stream until the end of the trial, at which point the encoded information can be passed on to response selection mechanisms, eventually leading to a response. While STC for Task $_{1}$ is taking place (STC ${ }_{1}$ in the figure), STC for Task ${ }_{2}$ is blocked. If $\mathrm{T}_{2}$ is masked, as it is in the RSVP paradigm used in Experiments 1-3, the representation of $T_{2}$ created or activated by $\mathrm{PE}$ mechanisms $\left(\mathrm{PE}_{2}\right.$ in the figure) begins to decay if it is not immediately subjected to STC $\left(\mathrm{STC}_{2}\right.$ in the figure). For more evidence suggesting the importance of masking $\mathrm{T}_{2}$, see Giesbrecht and Di Lollo (in press) and Jolicoeur (in press-b). For evidence of very poor memory for briefly identified objects, see Potter $(1976,1993)$.

We can equate $\mathrm{SE}$ and $\mathrm{PE}$ with Stage 1 processing in the two-stage model, the attentional dwell model, or Duncan's (1980) model. In the central interference theory, STC is the process of encoding information into STM. This stage is similar to Stage 2 processing in the two-stage model, the attentional dwell model, or Duncan's model. In these models, Stage 2 processing is required to make storage into STM possible. The role played by STC in the central interference theory and Stage 2 processing in the two-stage model or the attentional dwell model is similar. According to all three approaches, it is in this stage that there is a capacity limitation that leads to a decrease in accuracy in Task 2 . Because of this similarity, most results that the two-stage model or the attentional dwell model can explain are also explained by the central interference theory. There are differences across models, however, as already discussed. The central interference theory assumes that operations other than STC $_{1}$ can cause postponement of the $\mathrm{STC}_{2}$. This is one major difference between the central interference theory and either the two-stage model or the attentional dwell model. And it is this difference that allows the central interference theory 


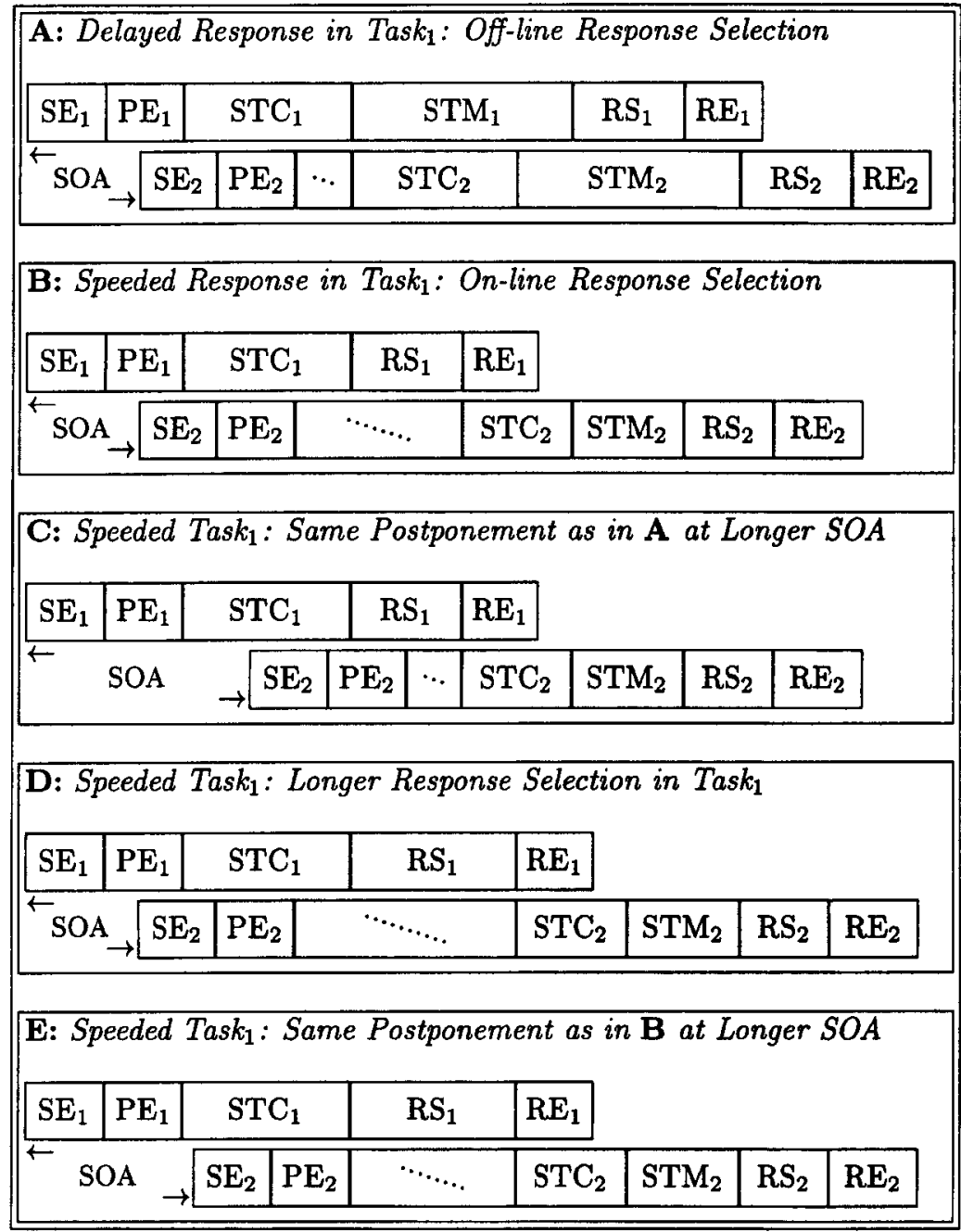

Figure 10. Model of task interactions. In each panel there are two stage diagrams. The top diagram illustrates the stages of processing mediating performance in Task ${ }_{1}$;

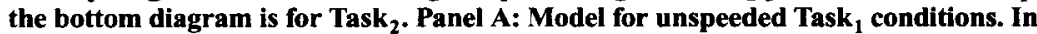
Task $_{1}$, following early encoding operations - sensory encoding $\left(\mathrm{SE}_{1}\right)$ and perceptual encoding ( $\left.\mathrm{PE}_{1}\right)$-the encoded information is subjected to short-term consolidation $\left(S_{1} C_{1}\right)$ processes. $S_{1 C}$ creates a copy of the information produced by $\mathrm{PE}_{1}$ in shortterm memory $\left(S T M_{1}\right)$. The information is maintained in STM until the end of the trial. At that point, response selection $\left(\mathrm{RS}_{1}\right)$ is performed, followed by response execution $\left(R_{1}\right)$. After a variable $S O A, T_{2}$ is presented (bottom diagram in Panel $\left.A\right)$. Following early encoding $\left(\mathrm{SE}_{2}\right.$ and $\mathrm{PE}_{2}$ ), the system would normally engage $\mathrm{STC}$ operations $\left(S_{T} C_{2}\right.$ ) to encode $T_{2}$ into STM. At short SOAs (as diagrammed here), however, $\mathrm{STC}_{2}$ cannot proceed because central mechanisms are busy carrying out $\mathrm{STC}_{1} . \mathrm{STC}_{2}$ must wait until $S T C_{1}$ terminates the $S T C$ of $T_{1}$. During this period of waiting, the representation of $T_{2}$ produced by $P E_{2}$ begins to decay (if $T_{2}$ was masked). Panel $B$ : Model for speeded Task conditions. In Task $_{1}$, after $\mathrm{STC}_{1}, \mathbf{R S}_{1}$ is performed, leading to $\mathbf{R E}_{1}$. $S C_{2}$ is postponed both by $S T C_{1}$ and $R_{1}$, leading to a longer period of central postponement than when only STC $_{1}$ needs to be performed, as is shown in Panel $A$. The longer postponement results in more decay of $T_{2}$, leading to worse recall. Panel $C$ : Model for speeded Task ${ }_{1}$ conditions, as in Panel $B$, but with $T_{2}$ presented at a longer SOA. Note that the same duration of $\mathrm{STC}_{2}$ postponement as that observed in Panel $\mathrm{A}$ is predicted by the model, but at the longer SOA. Panel D: Illustration of the consequences of a longer period of response selection in Task $\left(\operatorname{RS}_{1}\right)$ relative to the stage durations in Panel $\mathrm{B}$. A longer delay in $\mathrm{STC}_{2}$ results, for a given $\mathrm{SOA}$, producing a larger

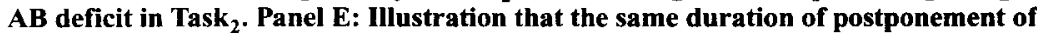
STC $_{2}$ produced in Panel B can be produced at a longer SOA if the duration of $\mathrm{RS}_{1}$ in Task $_{1}$ is longer. The AB effect should last longer. 
to explain the effects of Task ${ }_{1}$ response requirements on the magnitude of the $A B$ effect.

If the SOA between $T_{1}$ and $T_{2}$ is short, a period of waiting can occur after the masking of $\mathrm{T}_{2}$ and before the time at which STC for Task ${ }_{2}$ can begin (illustrated in Figure $10 \mathrm{~A}$ by declining dots before $\mathrm{STC}_{2}$ ). A longer period of waiting will generally be associated with a shorter SOA.

In Figure 10B, the effect of requiring an on-line response in Task $k_{1}$ is represented by assuming that response

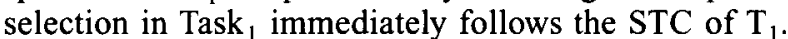
This added processing creates a longer period during which STC of $\mathrm{T}_{2}$ must wait, leading to more decay of the representation of $T_{2}$ and, thus, to a larger $A B$ effect.

For the sake of clarity, consider again Figure $10 \mathrm{~A}$ and Figure 10B. Note that the same SOA is assumed in both cases. At this constant SOA, the period of postponement of $\mathrm{STC}_{2}$ is longer when a speeded response was required

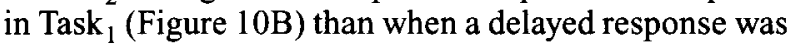
required (Figure 10A). A longer period of postponement of $\mathrm{STC}_{2}$ results in a greater loss of information about $\mathrm{T}_{2}$ (more decay). Thus, worse accuracy in Task ${ }_{2}$ is expected at this SOA with a speeded response than with a delayed response. That is, the $\mathrm{AB}$ effect should be larger (at least for intermediate SOAs).

Figure $10 \mathrm{C}$ illustrates that the $\mathrm{AB}$ effect should last longer when a speeded response is required. The same duration of postponement produced in Figure 10A can be obtained with a longer SOA. Thus, equivalent performance should be observed at these two SOAs, and hence a longer $\mathrm{AB}$ should result. This prediction was confirmed, but only for a limited range of SOAs (e.g., in Figure 4, compare performance in the unspeeded condition at 300 msec SOA with performance in the speeded condition at the 400-msec SOA).

At longer SOAs, however, better performance was found with a speeded Task $\mathrm{k}_{1}$ response than with a delayed response. At first blush, this finding may appear surprising and problematic for the model shown in Figure 10. It is likely, however, that the difference across conditions was caused by differences in the memory requirements

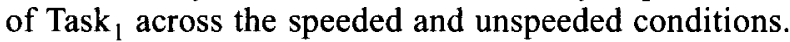
In the unspeeded condition, a memory representation of $T_{1}$ must be maintained in STM until after the end of the trial. In contrast, in the speeded condition, once the response has been selected, the representation of $T_{1}$ is no longer needed. This difference is illustrated in Figures $10 \mathrm{~A}$ and $10 \mathrm{~B}$. When Task ${ }_{1}$ is performed with a delayed response (Figure 10A), the processing of $\mathrm{T}_{2}$ must take place while a representation of $T_{1}$ is maintained in STM, which

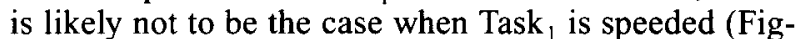
ure $10 \mathrm{~B}$ ). If some aspect of the processing of $\mathrm{T}_{2}$, such as $\mathrm{STC}_{2}$, is not as efficient when it is carried out with a concurrent load in STM (Logan, 1978), better performance would be expected in the speeded condition, but only when interference associated with on-line response selection had come to an end-that is, at longer SOAs.

Figure 10D illustrates the consequence of a longer period of response selection in Task ${ }_{1}$ than that shown in
Figure 10B: a longer period of postponement of $\mathrm{STC}_{2}$ results, leading to a larger $\mathrm{AB}$ effect. These postulated interactions are designed to account for the micro dependency between the magnitude of the $A B$ effect and the duration of $\mathrm{RT}_{1}$. They can also account for effects of direct manipulations of the duration of response selection in Task ${ }_{1}$ when $R_{1}$ is made on line (Jolicoeur, in press-a, in press-b). A similar effect would result also if the duration of STC $_{1}$ was lengthened (see Jolicoeur \& Dell'Acqua, 1996, 1998).

As $\mathrm{RT}_{1}$ lengthens, the $\mathrm{AB}$ should also last longer. This is illustrated by comparing Figure $10 \mathrm{E}$ with Figure 10B. The same duration of postponement of $\mathrm{STC}_{2}$ is shown in both cases, but it occurs at a longer SOA when $\mathrm{RT}_{1}$ is longer (panel E) than when $\mathrm{RT}_{1}$ is shorter (panel B). This prediction was confirmed, as can be seen in Figure 9. Note that comparisons of the effects of shorter versus longer $\mathrm{RT}_{1} \mathrm{~s}$ are made under equivalent memory load conditions, explaining why the prediction of both a larger and a longer $\mathrm{AB}$ is borne out.

It should also be noted that manipulations that would affect the duration of perceptual processing of $T_{1}$ should also produce effects on the magnitude and duration of the $\mathrm{AB}$ effect, according to the models shown in Figure 10. Consider Figure 10A and suppose that the duration of $\mathrm{SE}_{1}$ were lengthened by some experimental manipulation (e.g., by changing the contrast of $T_{1}$ in an RSVP paradigm, or by changing the masking effect of subsequent items). A longer period of $\mathrm{SE}_{1}$ would cause $\mathrm{STC}_{1}$ to begin later and, thus, to finish later. The later finishing time of $\mathrm{STC}_{1}$ means that $\mathrm{STC}_{2}$ could be postponed for a longer time, depending on just when the perceptual representation of $T_{2}$ became ready for STC. The observation here is that increasing the duration of $\mathrm{STC}_{1}$ itself or of any stage of processing before $S T C_{I}$ would likely lengthen the period of postponement of $\mathrm{STC}_{2}$, leading to a larger and longer $\mathrm{AB}$ effect. Thus, the fact that manipulations believed to have an effect on the perceptual processing of $T_{1}$ (such as different levels of masking) modulate the magnitude of the $A B$ effect is entirely consistent with the central interference theory. The present theory leads to the prediction that affecting the duration of $S E$ in Task $_{1}\left(\mathrm{SE}_{1}\right)$ would produce measurable effects in Task performance; yet $\mathrm{SE}_{1}$ per se does not interfere with any stage of processing required to perform Task ${ }_{2}$. Potential effects of manipulations of the duration of $\mathrm{SE}_{1}$ on accuracy in Task ${ }_{2}$ would all be mediated by changes in the onset and offset times of the operations of more central stages of processing, such as the STC of $\mathrm{T}_{1}\left(\mathrm{STC}_{1}\right)$. The foregoing remarks are intended to highlight the difficulties involved in pinpointing the likely locus (or loci) of dual-task interference producing the $\mathrm{AB}$ effect. The fact that a variable believed to affect the encoding of $T_{1}$ has effects in the AB paradigm does not, by itself, support the conclusion that $\mathrm{AB}$ is a perceptual phenomenon.

According to the central interference theory, the postponement of $\mathrm{STC}_{2}$ by $\mathrm{STC}_{1}$ and the postponement of $\mathrm{STC}_{2}$ by $\mathrm{RS}_{1}$ are two different causes of the AB effect 
because each of these processes $\left(\mathrm{STC}_{1}\right.$ and $\mathrm{RS}_{1}$ ) directly interferes with $\mathrm{STC}_{2}$. However, the theory allows us to provide a unified account of both effects by subsuming them as specific manifestations of a more general phenomenon: In both cases, the $\mathrm{AB}$ effect is a manifestation of the central postponement of STC ${ }_{2}$ by concurrent central processing required to perform Task ${ }_{1}$. It is this aspect of the theory that makes it similar to some accounts of the PRP phenomenon. In postponement models of the PRP effect (see, e.g., McCann \& Johnston, 1992), central interference is postulated at the level of RS: RS $_{1}$ is thought to postpone $\mathrm{RS}_{2}$. The central interference theory could provide the basis for a broad unification of the $A B$ and PRP phenomena by subsuming both as manifestations of central interference. However, it is clear that the two phenomena are not identical. According to the central interference theory, $\mathrm{AB}$ is produced by central interference of $\mathrm{STC}_{2}$, whereas PRP is likely produced by central interference of $\mathrm{RS}_{2}$. Therefore, it would not be surprising to discover that this difference could lead to observable empirical dissociations. Nonetheless, both AB and PRP could be similar in that they may both be manifestations of relatively late capacity limitations in the flow of information processing.

The results presented in this article, by themselves, do not provide unequivocal support for the model illustrated in Figure 10. Clearly, there are unresolved empirical and theoretical issues that will require additional research. For example, it is not clear whether STC must necessarily precede response selection when a speeded response

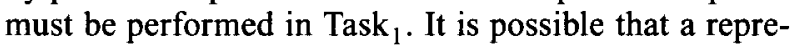
sentation of $T_{1}$ is made available to RS mechanisms via a late, capacity-demanding, stimulus categorization process (McCann \& Johnston, 1992). Perhaps this stage is required before either STC or RS, as suggested by Duncan (1980). Furthermore, this stage of processing, per se, could be capacity demanding and be involved in various dualtask interference phenomena. If STC $\mathrm{C}_{1}$ is not required for $\mathrm{RS}_{1}$, the models shown in Figures 10B-E will require amendment. The simplest amendment would be to replace the stages labeled $\mathrm{STC}_{1}$ and $\mathrm{RS}_{1}$ in the top stage diagrams in Figures $10 \mathrm{~B}-\mathrm{E}$ by a single stage, labeled $\mathrm{RS}_{1}$. More work will be required to disentangle this and other related issues.

This article reports evidence highlighting the importance of considering relatively late, or postperceptual mechanisms, such as RS, in explanations of the AB phenomenon. A new empirical technique made it possible to observe interactions with such postperceptual processes. The technique consisted of requiring an immediate re-

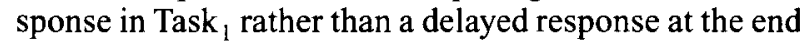
of the trial. Finally, a new theory was outlined, the central interference theory, which can account for observed effects of Task ${ }_{1}$ response requirements on the magnitude of the $A B$ effect and the dependence of $A B$ magnitude on $\mathrm{RT}_{1}$. The key assumption in this theory is that encoding information into STM involves a process-STC - that requires central mechanisms and that $\mathrm{STC}$ is susceptible to dual-task interference. The new theory may provide the basis for a broad unification of several presently nonintersecting lines of research, including the PRP effect, STM, the $A B$ phenomenon, and perhaps other closely related paradigms.

\section{REFERENCES}

Broadbent, D. E., \& Broadbent, M. H. P. (1987). From detection to identification: Response to multiple targets in rapid serial visual presentation. Perception \& Psychophysics, 42, 105-113.

CARrier, L. M., \& PAShler, H. (1995). Attentional limits in memory retrieval. Journal of Experimental Psychology: Learning, Memory, \& Cognition, 21, 1339-1348.

Chun, M. M., \& Potter, M. C. (1995). A two-stage model for multiple target detection in rapid serial visual presentation. Journal of Experimental Psychology: Human Perception \& Performance, 21, 109-127.

DE Jong, R., \& SWEET, J. B. (1994). Preparatory strategies in overlappingtask performance. Perception \& Psychophysics, 55, 142-151.

Duncan, J. (1980). The locus of interference in the perception of simultaneous stimuli. Psychological Review, 87, 272-300.

Duncan, J., Ward, R., \& Shapiro, K. L. (1994). Direct measurement of attentional dwell time in human vision. Nature, 369, 313-315.

GiesBrecht, B. L., \& Di Lollo, V. (in press). Beyond the attentional blink: Visual masking by item substitution. Journal of Experimental Psychology: Human Perception \& Performance.

JoliCOEUR, P. (in press-a). Concurrent response selection demands modulate the attentional blink. Journal of Experimental Psychology: Human Perception \& Performance.

JolicoevR, P. (in press-b). Dual-task interference and visual encoding. Journal of Experimental Psychology: Human Perception \& Performance.

Jolicoeur, P., \& Dell'Acqua, R. (1996, October). Attentional and structural constraints on short-term memory encoding. Paper presented at the 37th Annual Meeting of the Psychonomic Society, Chicago.

Jolicoevr, P., \& Dell'ACQUA, R. (1998). The demonstration of shortterm consolidation. Cognitive Psychology, 36, 138-202.

LofTus, G. F., \& Masson, M. E. J. (1994). Using confidence intervals in within-subject designs. Psychonomic Bulletin \& Review, 1, 476-490.

LogaN, G. D. (1978). Attention in character-classification tasks: Evidence for the automaticity of component stages. Journal of Experimental Psychology: General, 107, 32-63.

MCCANN, R. S., \& Johnston, J. C. (1992). Locus of the single-channel bottleneck in dual-task interference. Journal of Experimental Psychology: Human Perception \& Performance, 18, 471-484.

PASHLER, H. (1994). Dual-task interference in simple tasks: Data and theory. Psychological Bulletin, 116, 220-244.

PotTER, M. C. (1976). Short-term conceptual memory for pictures. Journal of Experimental Psychology: Human Learning \& Memory, 2, 509-522.

PotTer, M. C. (1993). Very short-term conceptual memory. Memory \& Cognition, 21, 156-161.

Potter, M. C., Chun, M. M., Banks, B. S., \& Muckenhoupt, M. (in press). Two attentional deficits in serial target search: The visual attentional blink and an amodal task-switch deficit. Journal of Experimental Psychology: Learning, Memory, \& Cognition.

Raymond, J. E., Shapiro, K. L., \& ARNELl, K. M. (1992). Temporary suppression of visual processing in an RSVP task: An attentional blink? Journal of Experimental Psychology: Human Perception \& Performance, 18, 849-860.

Raymond, J. E., Shapiro, K. L., \& Arnell, K. M. (1995). Similarity determines the attentional blink. Journal of Experimental Psychology: Human Perception \& Performance, 21, 653-662.

Rogers, R. D., \& MONSELL, S. (1995). Costs of a predictable switch between simple cognitive tasks. Journal of Experimental Psychology: General, 124, 207-231.

Ruthruff, E., Miller, J., \& Lachmann, T. (1995). Does mental rota- 
tion require central mechanisms? Journal of Experimental Psychology: Human Perception \& Performance, 21, 552-570.

SCARBorough, D. L. (1972). Memory for brief visual displays of symbols. Cognitive Psychology, 3, 408-429

SHAPIRO, K. L., \& RAYMOND, J. E. (1994). Temporal allocation of visual attention: Inhibition or interference? In D. Dagenbach \& T. H. Carr (Eds.), Inhibitory processes in attention, memory, and language (pp. 151-188). San Diego: Academic Press.

Shapiro, K. L., Raymond, J. E., \& ARNel L, K. M. (1994). Attention to visual pattern information produces the attentional blink in rapid serial visual presentation. Journal of Experimental Psychology: Human Perception \& Performance, 20, 357-371.

VAN Selst, M., \& Jolicoeur, P. (1994a). Can mental rotation occur before the dual-task bottleneck? Journal of Experimental Psychology: Human Perception \& Performance, 20, 905-921.

VAN SELST, M., \& Jolicoeur, P. (1994b). A solution to the effect of sample size on outlier elimination. Quarterly Journal of Experimental Psychology, 47A, 631-650.

Ward, R., DunCan, J., \& Shapiro, K. L. (1996). The slow time-course of visual attention. Cognitive Psychology, 30,79-100.

Weichselgartner, E., \& Sperl.ING, G. (1987). Dynamics of automatic and controlled visual attention. Science, 238, 778-780.
Wolfe, J. M. (1994). Guided Search 2.0: A revised model of visual search. Psychonomic Bulletin \& Review, 1, 202-238.

\section{NOTE}

1. The data in each cell are sorted, and the most extreme observation is temporarily excluded from consideration. The mean and standard deviation of the remaining numbers is then computed. Cutoff values are established using the following equations:

$$
V_{\text {low }}=\bar{X}-C * S D \quad V_{\text {high }}=\bar{X}+C * S D .
$$

The smallest and largest observation in the cell are then checked against the cutoff values, $V_{\text {low }}$ and $V_{\text {high }}$. If one or both are outside the bounds, they are defined as outliers and excluded from further consideration. If an outlier is found, the algorithm is applied anew to the remaining data The value of $C$ depends on the sample size such that the estimated final mean is not influenced by sample size (see Van Selst \& Jolicoeur, 1994b).

(Manuscript received February 26, 1997; revision accepted for publication November 24,1997 .) 